\title{
Natural Resource Management: A Network Perspective
}

\author{
Efthymia Kyriakopoulou $^{1}\left(\mathbb{D} \cdot\right.$ Anastasios Xepapadeas $^{2}$
}

Accepted: 27 June 2021 / Published online: 19 August 2021

(c) The Author(s) 2021

\begin{abstract}
This paper studies the role of social networks in the management of natural resources. We consider a finite number of agents who exploit a specific natural resource. Harvesting is subject to three external effects, namely resource stock externalities, crowding externalities, and collaboration spillovers. We show that the structure of the social network-defined by the presence of collaboration links between individual agents - determines the equilibrium and the optimal harvesting amount. We then allow the agents to make decisions about creating or eliminating cooperation links, which endogenizes the structure of the network and is proved to affect total harvesting and aggregate welfare. Conservation plans are shown to change the regulator's objective and increase even further the gap between the decentralized and the optimal outcomes. We show that the optimal policy depends explicitly on the structure of the network and the 'centrality' of the associated agents. Finally, introducing heterogeneity is proved to affect both individual profits and the incentives to create cooperation links.
\end{abstract}

Keywords Environmental externalities $\cdot$ Social networks · Natural resource management · Optimal network structure

JEL Classification D85 · H23 · Q30

\begin{abstract}
We thank the co-editor, Mireille Chiroleu-Assouline, and two anonymous reviewers for their helpful comments on a previous version of this paper. We also thank Katrin Millock, Alessandro Tavoni, Yves Zenou, and participants at the 6th World Congress of Environmental and Resource Economists, the 19th Annual BioEcon Conference, and the 2018 SURED Conference for helpful comments and discussions.
\end{abstract}

Efthymia Kyriakopoulou

Efthymia.kyriakopoulou@slu.se

Anastasios Xepapadeas

xepapad@aueb.gr

1 Department of Economics, Swedish University of Agricultural Sciences, Box 7013, 75007 Uppsala, Sweden

2 Department of International and European Economic Studies, Athens University of Economics and Business and Department of Economics, University of Bologna, Bologna, Italy 


\section{Introduction}

Resource appropriators are usually not capable of designing rules that secure the sustainable use of the resource. However, they are all jointly affected by everything each one of them is doing. Resource use is so interconnected that all agents should take into account the choices of the rest of the agents when taking individual decisions. In the context of natural resources, this intragroup externality may imply both positive and negative effects. For example, if a fisher occupies a good fishing site, the second fisher arriving at the same location will have to find another site. If, however, the two fishers coordinate, they can agree in advance on who will occupy this site and who will move to another site. Along the same lines, when a fisher allocates time and material to improving some fishing area, those sites will be used by other fishers who will also enjoy the effort and money spent by the first user.

In this paper, we use a resource model that takes into account these intragroup externalities and analyzes the resulting dependence of individual outcomes on group behavior. This dependence of individual outcomes on group behavior is known as "peer effects" in the literature of social networks. Social network models are used to analyze non-market interactions. Thus, environmental and resource management issues-where externalities play a central role-can be clearly considered in the context of a social network. The nodes of the network could be polluting firms, or firms adopting clean technologies, agents harvesting an exhaustible or a renewable resource, countries emitting greenhouse gases and adopting mitigation or adaptation policies, or consumers engaging in polluting or pollution-reducing activities.

A particular characteristic of environmental networks is the fact that these networks can be characterized by strategic heterogeneity. That is, the network includes both strategic complementarities, emerging when the marginal payoff of an agent is increasing in the actions of her neighbors, and strategic substitutabilities, when the marginal payoff of an agent is decreasing in the actions of her neighbors. The first case may emerge in links indicating cost-reducing technology agreements and cooperation among agents. Examples of strategic substitutability could be the case of congestion effects, or increasing search or extraction costs when the stock of a resource exploited by the network is depleted. The study of this potential heterogeneity in a network context is shown to provide new insights in terms of market outcomes and policies to attain the social optimum.

In this paper, we examine how the creation of collaboration links between resource appropriators affects the exploitation of the resource. The presence or not of a collaboration link between all the possible pairs of agents will determine the network structure. The key fact here is that resource users are tied together as long as they continue to share the same resource. As pointed out by Ostrom (1990) in her seminal analysis about collective action in common pool resources (CPR), when appropriators exploit a CPR independently (Fig. 1b), the total benefit obtained is lower compared to what they would achieve if they had decided to coordinate their strategies (Fig. 1a). This can lead either to lower returns or, in the worst case, in the destruction of the CPR. Thus, collective action is important in achieving the highest possible joint return. ${ }^{1}$

\footnotetext{
1 Collective action does not need to be associated with formal organizations. As pointed out in Ostrom (2004), collective action can be informal, where local networks or local groups organize and coordinate local action in order to achieve specific purposes.
} 


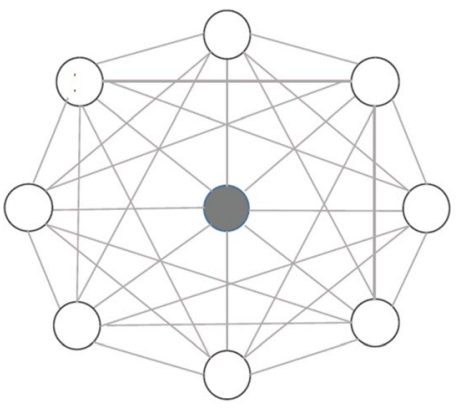

(a)

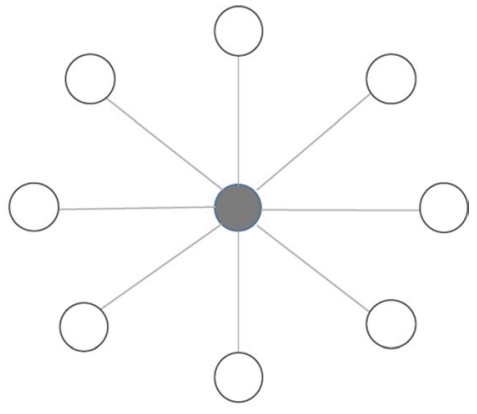

(b)

Fig. 1 White nodes: resource appropriators. Gray node: resource. a Collective action, b Independent action

The different results under collective, subgroup or independent action are analyzed and policies that take into account the specific network structure are designed so as to promote the formation of the optimal network structure. This is particularly important in a CPR problem since the problem that CPR appropriators face is how to organize. In other words, how to change the situation where appropriators act independently to a new one, where they adopt a more collective action approach and enjoy higher joint benefits while at the same time reducing their joint harm. Formally, in this paper we study stable network structures in equilibrium, as well as at the optimum, and determine policies that can close the gap between the optimal and the market network structures.

More specifically, in this paper we consider a network consisting of a finite number of agents located at distinct spatial locations who exploit a depletable resource which is located at a site different than the locations of the agents. The exploited quantity of the resource is subject to three external effects: (1) Resource stock externalities, which occur when total private cost increases when resource stock decreases, (2) Crowding externalities, which imply that cost is increasing in the harvesting of co-appropriators, and (3) collaboration or knowledge spillovers, which occur when agents are involved in some kind of collaboration activities by coordinating with the rest of the agents and thereby decreasing the negative effect of crowding externalities. ${ }^{2}$ The existence of externalities implies that the market outcome differs from the optimal one. More precisely, in equilibrium the involved agents maximize their private profits. On the contrary, at the optimum, the social planner maximizes the private value of the network, which is the sum of the agents' payoffs, but at the same time she also takes into account the scarce nature of the resource by explicitly considering its conservation value. This could facilitate the discussions regarding conservation versus development (e.g. Scullion et al. 2016; Delgado-Serrano 2017), which are based on the fact that natural resources should not be treated in the same way as producible goods. Therefore one of the contributions of this paper is to show how the network structure and the associated payoffs are affected by this special nature of natural resources.

Congestion externalities are assumed to affect all users, regardless of whether they collaborate or not, and increase with the number of appropriators. The creation of collaboration links between the appropriators is represented by a network: a link between two

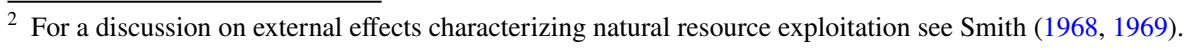


appropriators indicates that those two agents collaborate and agree on specific strategies that have to be followed when exploiting the resource. This can also be interpreted as efforts to reduce the magnitude of the congestion externality. Taking turns to exploit the resource, or agreeing on who will occupy specific harvesting sites, for example, requires some minimum collaboration among the agents, but at the same time, it reduces congestion. The two opposing effects are illustrated in Fig. 1. In Fig. 1b, we can see the effect of congestion externalities: the more users exploit the resource, the higher the implied congestion. In Fig. 1a, we can observe the case where all appropriators collaborate with each other. So apart from the fact that they all exploit the same resource (as in Fig. 1b), they also collaborate with each other, which results in a complete network structure.

This paper uses recent results from the theory of social networks (e.g. Ballester et al. 2006; Helsley and Zenou 2014; Verdier and Zenou 2017) to study the impact of social interactions among resource users on the exploitation of natural resources. The need to incorporate social network analysis in this literature has been recently highlighted and discussed in related work (e.g. Carlsson and Sandström 2008; Bodin and Prell 2011). More precisely, collaboration among resource users has been shown to facilitate the spread and sharing of ecological knowledge within their community (Crona and Bodin 2006). Trust and cooperation between individual agents are important for the promotion of sustainable practices in natural resource management (Gray et al. 2012). However, heterogeneityin terms, for example, of ethnic diversity-among individuals prevents the formation of social ties and creates challenges for collaboration across groups (Pomeroy et al. 2007; Barnes et al. 2013). Thus, effective management requires the consideration not only of the biological and ecological characteristics of natural resources, but also of the social aspect of the system (Bodin and Prell 2011; Barnes et al. 2013). Experiments and qualitative field studies have confirmed the important role of social networks in the sustainable management of natural resources. ${ }^{3}$ To the best of our knowledge, this is the first paper that uses the tools of the theory of social networks to analyse the impact of social interactions among resource users on the exploitation of a natural resource.

In this context, we study the market outcomes associated with specific network structures and eventually characterize the most efficient market structure. We also characterize the socially optimal network structure and examine policies under which market outcomes will reproduce the social optimal structure. Our network game has an interior Nash equilibrium that is proportional to the Bonacich network centrality. Using this Bonacich measure allows us to express the relation between the equilibrium strategic behavior and the network topology. More precisely, we show that the individual agent's equilibrium outcome is related to the player's position in the local interactions network. We also show that the aggregate equilibrium effort increases with the density and with the number of local interactions.

Our results have important policy implications. The main advantage and contribution of the network approach is that by identifying the efficient structure, desirable or non-desirable

\footnotetext{
${ }^{3}$ Crona and Bodin (2006) study the social interactions among fishermen in a coastal seascape in Kenya and show that communication occurs only between fishermen who use the same gear type, which prevented the exchange of ecological knowledge within the community. In a similar framework, Bodin and Crona (2008) show that homogeneity of the resource users and the social ties formed among them contribute to the successful management of natural resources. In a more recent study, Shreedhar et al. (2020) examine experimentally how the different relationships between group members (presented by complete or imperfect networks) can deter or promote over-appropriation depending on the possibilities to coordinate and punish the non-cooperative behaviour.
} 
links among the agents can be determined and policies can therefore be designed not just to control the level of externalities but also to control for the desired link structure among agents under strategic complementarities and substitutabilities. Disregarding the structure of the network-when this structure affects individual and aggregate profits-is shown to result in inefficient policies.

The rest of the paper is organized as follows. The next section presents the related literature. Section 3 presents the model and solves for the market outcome. Section 4 solves for the optimum, while in Sect. 5, we describe the dynamics of network formation. In Sect. 6 we apply the theory in a small numbers network. Section 7 extends the model by introducing heterogeneity and the final section concludes the paper.

\section{Related Literature}

In order to study the dependence of individual outcomes on group behavior, we use the tools and the theory of social networks. Helsley and Zenou (2014) and Verdier and Zenou (2017) study how social interactions among individuals in a particular community affect their choice of geographic location or their education effort (strategic complementarity). Ballester et al. (2006) and Jackson and Zenou (2015) model network games that are characterized by both local complementarities and global competitive effects. For overviews of how the network theory has been applied in different fields of economics see Ioannides (2012), Jackson (2014), Jackson and Zenou (2015) and Jackson et al. (2017).

Despite the critical importance of social networks on environmental issues, network theory has been neglected in environmental and resource economics literature. There are, though, some exceptions of recent theoretical contributions that include more explicit network structures in their analysis. In this context, Conley and Udry (2001) study the role of social networks on the adoption of innovative, environmentally-friendly technologies by firms and show that high network density leads to the development of new technology and the diffusion of more sustainable management practices. In a more recent paper, Günther and Hellmann (2017) study the stability of International Environmental Agreements (IEA) when pollution has both a global and a local effect, where local pollution spillovers are represented by a network structure. Their main finding is that for stable IEAs to exist, the network structure needs to be balanced.

Closer to the present study is İlkiliç (2011), who uses a network model of common property resources with multiple sources and users to study how the exploitation of each source by a different number of users, as well as the connection of each user with one or more sources, leads to different levels of extraction by users and outflows from sources. Contrary to our paper where the network represents the collaboration links between the different appropriators, in İlkiliç (2011) the links connect users to a different number of sources. In other words, İlkiliç (2011) studies the right of different users to exploit a different number of sources, while here, we explore the incentives of appropriators to collaborate with the rest of the users and show how denser networks affect aggregate harvesting and aggregate profits.

Barnes et al. (2016a) study how the formation of social networks affects fishers' behavior and actions and show that fishers form subgroups with people of the same ethnicity, which is strongly correlated with shark bycatch. In particular, their analysis suggests that enhanced communication across segregated groups could have prevented the incidental catch of over 46,000 sharks between 2008 and 2012 in a single commercial fishery. Their 
results suggest that having a better understanding of social interactions across resource users groups can lead to more sustainable environmental outcomes. Network analysis can thus shed more light on the interdependence of resource appropriators by analyzing the incentives of individual users to connect with the other users or not.

Recent contributions in the public good and natural resource management literature show that collective action can succeed. Experimental and empirical studies have highlighted the role of social integration and cooperation among resource users (e.g. Cavalcanti et al. 2010, 2013; Tembata and Takeuchi 2018). These studies show that individual agents can sustain collective action without the involvement of any governmental organization. Public good experiments indicate that the decision of an individual to collaborate depends on the level of collaboration of others (Fischbacher et al. 2001). Thus, the presence of collaboration links between resource users is important. In the same context, a growing theoretical literature explores the role of networks in the provision of public goods (e.g. Bramoullé and Kranton 2007; Allouch 2015) which has important implications for the successful cooperative self-governance of natural resources.

For a broad discussion on how networks can be used in order to facilitate the study of environmental issues see Currarini et al. (2016). The authors explain that the application of network economics to environmental and resource issues is still in its infancy and there are a lot of issues that need to be explored by using this new approach.

\section{Cake-Eating and Competitive Exploitation in a Network}

\subsection{Nash Equilibrium and Bonacich Centrality}

We consider a depletable resource of fixed stock $S$ that is exploited by $n$ agents. Exploitation lasts one period and harvest depends on the amount of effort, $E$, that is made during harvesting and on the size of the resource stock, $S$. Then, the harvest function is given by:

$$
H(E, S)=q E S
$$

where $q$ is the "catchability-coefficient," which can translate one unit of effort into one unit of harvest. Solving (1) with respect to $E$ will give us:

$$
E_{i}=\frac{H_{i}}{q S}
$$

When the resource is more scarce, the agents need to put more effort into harvesting it, which increases the private cost of harvesting $\left(\frac{1}{2} \beta E_{i}^{2}\right)$. Thus, the pay-off of agent $i$ will be:

$$
u_{i}=\underbrace{p H_{i}-\frac{1}{2} \frac{\beta}{(q S)^{2}} H_{i}^{2}}_{\text {"own" net profit }}-\underbrace{\gamma \sum_{j \neq i}^{n} H_{i} H_{j}}_{\text {global interaction effect }}+\underbrace{\delta \sum_{j=1}^{n} g_{i j} H_{i} H_{j}}_{\text {local interaction effect }},
$$

where $p$ is the price of the resource which is taken as exogenous and $\beta$ is a parameter associated with the private cost of harvesting. Along with the "own" net benefits of harvest, appropriators have to consider two more effects: the global interaction effect and the local interaction effect. The first one is interpreted as a global substitutability effect, which creates some type of congestion. The more the rest of the agents exploit the resource, the 
lower my benefit is. The second effect reflects the local complementarity component. ${ }^{4}$ That is, collaboration links between the agents decrease the magnitude of the congestion externality, since agents, for example, could coordinate and agree to take turns when exploiting the resource. ${ }^{5}$ The marginal impacts of the global and the local interaction effect are denoted by $\gamma$ and $\delta$, respectively. In a resource context, it is more probable to have $\gamma>\delta$, meaning that the congestion effect dominates the positive externality. However, it is possible to have (and interesting to explore) the case where $\delta>\gamma$, which implies that collaboration outweighs congestion.

We set $g_{i i}=0$ and $0 \leq g_{i j} \leq 1$, for $i \neq j$. For simplicity, $g_{i j}$ will be either 0 when there is no link between nodes $i$ and $j$, or 1 when agents $i$ and $j$ decide to collaborate. The maximization problem can be written as:

$$
\begin{aligned}
& \max _{H_{i}} u_{i} \\
& \text { subject to } \sum_{j=1}^{n} H_{j} \leq S, H_{j} \geq 0 .
\end{aligned}
$$

In "Appendix A1", we present the conditions that should be satisfied in order to have interior solutions. The equilibrium harvesting solves:

$$
H_{i}^{*}=\frac{p-\gamma \sum_{j=1}^{n} H_{j}^{*}+\delta \sum_{j=1}^{n} g_{i j} H_{j}^{*}}{\frac{\beta}{q^{2} S^{2}}-\gamma} .
$$

We will now define the Bonacich network centrality measure that will be used in the equilibrium analysis. ${ }^{6}$ For this purpose, we will use the n-square adjacency matrix $\mathbf{G}$ that keeps track of all the direct connections in the network $\mathbf{g}$. Thus, $g_{i j}$ will show if agents $i$ and $j$ are directly connected $\left(g_{i j}=1\right)$ or not $\left(g_{i j}=0\right)$. In order to take into account the indirect connection, we need to define the matrix $\mathbf{G}^{k}$, which is the $k$ th power of $\mathbf{G}$, with coefficient $g_{i j}^{[k]}$, where $k$ is some integer. More specifically, $g_{i j}^{[k]} \geqslant 0$ measures the number of paths of length $k \geq 1$ in $\mathbf{g}$ from $i$ to $j$. We can then define the matrix

$$
\mathbf{M}(\mathbf{g}, \hat{\delta})=[\mathbf{I}-\hat{\delta} \mathbf{G}]^{-1}=\sum_{k=0}^{+\infty} \hat{\delta}^{k} \mathbf{G}^{k}
$$

where $\hat{\delta} \geq 0$ is a scalar that takes low enough values and $m_{i j}(g, \hat{\delta})=\sum_{k=0}^{+\infty} \hat{\delta}^{k} g_{i j}^{[k]}$ are the elements of the matrix. ${ }^{7}$ The Bonacich centrality of network $\mathbf{g}$ is $\mathbf{b}(\mathbf{g}, \hat{\delta})=[\mathbf{I}-\delta \mathbf{G}]^{-1} \cdot \mathbf{1}$. The Bonacich centrality of node $i$ is then $b_{i}(\mathbf{g}, \hat{\delta})=\sum_{j=1}^{n} m_{i j}(\mathbf{g}, \hat{\delta})$, which is the sum of all selfloops and all the other loops, i.e., $b_{i}(\mathbf{g}, \hat{\delta})=m_{i i}(\mathbf{g}, \hat{\delta})+\sum_{\substack{j \neq 1 \\ j \neq i}}^{n} m_{i j}(\mathbf{g}, \hat{\delta})$.

\footnotetext{
${ }^{4}$ For examples of modelling positive interactions in network models, see Jackson and Zenou (2015).

${ }^{5}$ Here, we assume that $\delta$ captures the collaboration benefits, net of collaboration costs. Alternatively, we could explicitly assume that the creation of collaboration links is costly and the cost to create or keep a collaboration link is equal to $c$. In this case, net collaboration benefits would be given by: $\delta-c$.

${ }^{6}$ The Bonacich centrality (Bonacich 1987) measures the importance of the number of direct and indirect connections that an agent has with the rest of the agents.

$7 \hat{\delta}$ gives relatively lower weight to longer paths. The coefficients $m_{i j}(g, \hat{\delta})=\sum_{k=0}^{+\infty} \hat{\delta}^{k} g_{i j}^{[k]}$ of the $\mathbf{M}(\mathbf{g}, \hat{\delta})$ matrix count the number of paths that start from $i$ and end in $j$, while the paths of length $k$ are weighted by $\hat{\delta}^{k}$.
} 
Let us assume that $\lambda_{1}(\mathbf{G})$ is the largest eigenvalue of $\mathbf{G}$, with $\lambda_{1}(\mathbf{G})>0$. Then if $\frac{\beta}{q^{2} S^{2}}-\gamma>\delta \lambda_{1}(\mathbf{G})$, we can show that there is a unique Nash equilibrium. ${ }^{8}$ We first define the square matrix of aggregate cross-effects: $\boldsymbol{\Sigma}=\left[\sigma_{i j}\right]$, as follows: $\boldsymbol{\Sigma}=-\frac{\beta-\gamma q^{2} S^{2}}{q^{2} S^{2}} \mathbf{I}-\gamma \mathbf{U}+\delta \mathbf{G}$, where $\mathbf{U}$ denotes the n-square matrix of ones. An interior Nash equilibrium in pure strategies $\mathbf{H}^{*} \in \mathbb{R}_{+}^{n}$ is such that $\frac{\partial u_{i}}{\partial H_{i}\left(\mathbf{H}^{*}\right)}=0$ and $H_{i}^{*}>0$ for all $i=1, \ldots, n$. If such an equilibrium exists, then it solves:

$$
-\boldsymbol{\Sigma} \cdot \mathbf{H}=\left[\frac{\beta-\gamma q^{2} S^{2}}{q^{2} S^{2}} \mathbf{I}+\gamma \mathbf{U}-\delta \mathbf{G}\right] \cdot \mathbf{H}=p \cdot \mathbf{1} .
$$

Let $\hat{\delta}=\frac{\delta q^{2} S^{2}}{\beta-\gamma q^{2} S^{2}}$. Solving for $\mathbf{H}$ and using the Bonacich centrality measure, we get the Nash equilibrium harvesting $\mathbf{H}^{*}$ :

$$
\mathbf{H}^{*}(\boldsymbol{\Sigma})=\frac{p}{\frac{\beta-\gamma q^{2} S^{2}}{q^{2} S^{2}}+\gamma b\left(\mathbf{g}, \frac{\delta q^{2} S^{2}}{\beta-\gamma q^{2} S^{2}}\right)} \mathbf{b}\left(\mathbf{g}, \frac{\delta q^{2} S^{2}}{\beta-\gamma q^{2} S^{2}}\right),
$$

where $b\left(\mathbf{g}, \frac{\delta q^{2} S^{2}}{\beta-\gamma q^{2} S^{2}}\right)$ is the sum of the unweighted Bonacich centralities of all players:

$$
\begin{aligned}
& b\left(\mathbf{g}, \frac{\delta q^{2} S^{2}}{\beta-\gamma q^{2} S^{2}}\right)=b_{1}\left(\mathbf{g}, \frac{\delta q^{2} S^{2}}{\beta-\gamma q^{2} S^{2}}\right) \\
& \quad+b_{2}\left(\mathbf{g}, \frac{\delta q^{2} S^{2}}{\beta-\gamma q^{2} S^{2}}\right)+\cdots+b_{n}\left(\mathbf{g}, \frac{\delta q^{2} S^{2}}{\beta-\gamma q^{2} S^{2}}\right) .
\end{aligned}
$$

The Nash equilibrium harvesting of agent $i$ is:

$$
H_{i}^{*}(\mathbf{g})=\frac{p}{\frac{\beta-\gamma q^{2} S^{2}}{q^{2} S^{2}}+\gamma b\left(\mathbf{g}, \frac{\delta q^{2} S^{2}}{\beta-\gamma q^{2} S^{2}}\right)} b_{i}\left(\mathbf{g}, \frac{\delta q^{2} S^{2}}{\beta-\gamma q^{2} S^{2}}\right),
$$

which is proportional to her Bonacich centrality in the social network. This leads to the following proposition:

Proposition 1 (Equilibrium harvesting) For any network $\mathbf{g}$, if $\delta \lambda_{1}(\mathbf{g})<\frac{\beta}{q^{2} S^{2}}-\gamma$ and $S>\frac{p}{\gamma-\delta}$, there exists a unique, interior Nash equilibrium in harvesting in which the harvesting amount by any agent $i$ (given by (10)) is proportional to her Bonacich centrality.

Proof The conditions for interior solution can be found in "Appendix A1" and the full derivation of the equilibrium in "Appendix A2".

The Nash equilibrium harvesting $H_{i}^{*}(\mathbf{g})$ depends on individual $i$ 's position in the social network, i.e. on the number of collaboration links she forms with the rest of the resource users. Expression (10) shows that more connected individuals extract a higher quantity of

\footnotetext{
8 The condition $\frac{\beta}{q^{2} S^{2}}-\gamma>\delta \lambda_{1}(\mathbf{G})$ requires that local complementarities are small enough compared to own concavity, that prevents multiple equilibria and also guarantees that $\left[\left(\frac{\beta}{q^{2} S^{2}}-\gamma\right) \mathbf{I}-\delta \mathbf{G}\right]$ is invertible (see Ballester et al. 2006).

9 The derivation of the equilibrium harvesting can be found in "Appendix A2".
} 


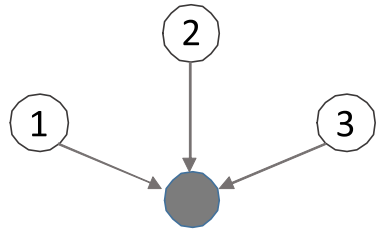

(a)

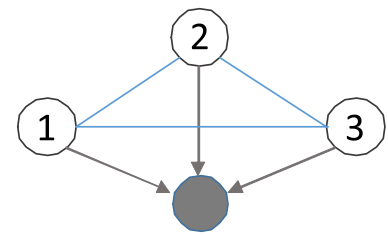

(b)

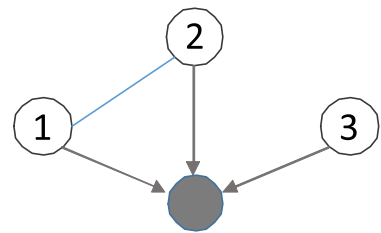

(c)

Fig. 2 A 3-agent resource network

the resource, compared to less connected agents. Intuitively, it is easier for more connected resource users to coordinate with their neighbors, take turns to extract the resource and avoid congestion. ${ }^{10}$ It is easy to show that $H_{i}^{*}(\mathbf{g})$ increases with the price of the resource, $p$, and the aggregate resource stock, $S$, but decreases when the private cost of extraction, $\beta$, and the negative effect of congestion, $\gamma$, increase. The role of $\delta$ is discussed in Proposition 3.

Let us now discuss how the density of the network affects aggregate resource use. Assume that there are two symmetric networks, $\mathbf{g}$ and $\mathbf{g}^{\prime}$, that include the same individuals. Assume, also, that all the agents who are connected in $\mathbf{g}$ are also connected in $\mathbf{g}^{\prime}$, but there is at least one pair of agents (say $m+1$ and $m+1$ ) who are directly connected in $\mathbf{g}^{\prime}$, but not in $\mathbf{g}$, i.e. $\mathbf{g}_{m, m+1}^{\prime}=1$ and $\mathbf{g}_{m, m+1}=0$. Then, we show in Proposition 2 that aggregate resource use is higher in $\mathbf{g}^{\prime}$ than in $\mathbf{g}$.

Proposition 2 (Aggregate harvesting) Let $\mathbf{g}$ and $\mathbf{g}^{\prime}$ be symmetric such that $\mathbf{g}^{\prime} \geq \mathbf{g}$. Then, $\sum_{i} H_{i}^{*}\left(\mathbf{g}^{\prime}\right)>\sum_{i} H_{i}^{*}(\mathbf{g})$, if $\delta \lambda_{1}(\mathbf{g})<\frac{\beta}{q^{2} S^{2}}-\gamma$ and $\delta \lambda_{1}\left(\mathbf{g}^{\prime}\right)<\frac{\beta}{q^{2} S^{2}}-\gamma$.

Proof The proof is available in "Appendix A3" where we show that a more dense network leads to higher aggregate harvesting. This is because in a more dense network, appropriators form more collaboration links with their competitors, which allows them to reduce the magnitude of the congestion effect. Thus, market efficiency leads to higher aggregate harvesting in $\mathbf{g}^{\prime}$ than in $\mathbf{g}$.

\subsection{Examples}

\subsubsection{A 3-Agent Star Network}

To illustrate our results, we continue with a simple example of three unconnected agents who exploit the resource, as illustrated in Fig. 2a. Here, we assume that there is no local interaction effect, meaning that resource appropriators act independently. The arrows in Fig. 2 show that all three agents are exploiting the same resource, creating some congestion externality.

Notice that since the agents are not connected, the adjacency matrix $\mathbf{G}$ is a $(3 \times 3)$ zero matrix, so $\mathbf{M}=\mathbf{M}^{-1}=\mathbf{I}$ and the Bonacich centrality vector is the vector of ones $\mathbf{b}=(1,1,1)$. Using (10), we can now compute the agents' harvesting amounts (with $b=3)$ :

${ }^{10}$ Here taking turns does not imply a dynamic framework, as the sequence of extraction does not matter. 


$$
H_{i}^{*}=\frac{p q^{2} S^{2}}{\beta+2 \gamma q^{2} S^{2}}
$$

In the case where there is no congestion, or in the case of a single user, price equals private marginal cost, $p=\beta\left(\frac{H_{i}}{q^{2} S^{2}}\right)$, which leads to $H_{i}^{N C}=\frac{p q^{2} S^{2}}{\beta}$. It is easy to see that congestion makes harvesting more costly, which leads to a reduction in the equilibrium amount of harvesting:

$$
\frac{d H_{i}^{*}}{d \gamma}=-\frac{2 p q^{4} S^{4}}{\left(\beta+2 \gamma q^{2} S^{2}\right)^{2}}<0
$$

Thus, $H_{i}^{*}<H_{i}^{N C}$, for any $\gamma>0$.

Individual profits under the presence of this negative externality are given by:

$$
u_{i}^{*}=\frac{p^{2} q^{2} S^{2} \beta}{2\left(\beta+2 \gamma q^{2} S^{2}\right)^{2}}
$$

while aggregate profits are given by:

$$
u_{T}^{*}=\frac{3 p^{2} q^{2} S^{2} \beta}{2\left(\beta+2 \gamma q^{2} S^{2}\right)^{2}}
$$

\subsubsection{A 3-Agent Full Network}

Let us now analyze the case illustrated in Fig. $2 \mathrm{~b}$ above, where all agents are linked to each other, creating collaboration links. The adjacency matrix of this network is given by:

$$
\mathbf{G}=\left[\begin{array}{lll}
0 & 1 & 1 \\
1 & 0 & 1 \\
1 & 1 & 0
\end{array}\right]
$$

and the corresponding Bonacich centralities are given by the vector:

$$
\mathbf{b}=\left(\frac{\beta-\gamma q^{2} S^{2}}{\beta-(\gamma+2 \delta) q^{2} S^{2}}, \frac{\beta-\gamma q^{2} S^{2}}{\beta-(\gamma+2 \delta) q^{2} S^{2}}, \frac{\beta-\gamma q^{2} S^{2}}{\beta-(\gamma+2 \delta) q^{2} S^{2}}\right)
$$

Harvesting for each agent is symmetric and given by:

$$
\widehat{H}_{i}=\frac{p q^{2} S^{2}}{\beta+2(\gamma-\delta) q^{2} S^{2}}
$$

It is easy to see that $\widehat{H}_{i}>H_{i}^{*}$, meaning that the fully connected network leads to higher use of the resource than the unconnected network. Individual equilibrium payoffs are given by:

$$
\hat{u}_{i}=\frac{p^{2} q^{2} S^{2} \beta}{2\left(\beta+2(\gamma-\delta) q^{2} S^{2}\right)^{2}}
$$


while aggregate profits are given by:

$$
\hat{u}_{T}=\frac{3 p^{2} q^{2} S^{2} \beta}{2\left(\beta+2(\gamma-\delta) q^{2} S^{2}\right)^{2}}
$$

We can now illustrate Proposition 2 by considering the aggregate harvesting, $H_{T}^{*}=\sum_{i=1}^{3} H_{i}^{*}$, and profits of the star network (14) and the corresponding ones of the full network, $\widehat{H}_{T}=\sum_{i=1}^{3} \widehat{H}_{i}$, and (18). Not surprisingly, $\hat{H}_{T}>H_{T}^{*}$ and $\hat{u}_{T}>u_{T}^{*}$, which confirms the fact that denser networks generate more aggregate profits that correspond to a higher harvesting amount than less dense networks.

\subsubsection{Partly Connected Network: Two Types of Agents}

Lastly, we study the case illustrated in Fig. 2c where two of the agents (say 1 and 2) create a collaboration link while the third agent acts independently. The adjacency matrix $\mathbf{G}$ in this case is:

$$
\mathbf{G}=\left[\begin{array}{lll}
0 & 1 & 0 \\
1 & 0 & 0 \\
0 & 0 & 0
\end{array}\right]
$$

and the vector of Bonacich centralities of the three players is:

$$
\mathbf{b}=\left(\frac{\beta-\gamma q^{2} S^{2}}{\beta-(\gamma+\delta) q^{2} S^{2}}, \frac{\beta-\gamma q^{2} S^{2}}{\beta-(\gamma+\delta) q^{2} S^{2}}, 1\right)
$$

Harvesting for symmetric agents 1 and 2 is:

$$
\tilde{H}_{1,2}=\frac{p q^{2} S^{2}\left(\beta-\gamma q^{2} S^{2}\right)}{\beta^{2}+(\gamma-\delta) \beta q^{2} S^{2}-2 \gamma^{2} q^{4} S^{4}}
$$

while for agent 3 :

$$
\tilde{H}_{3}=\frac{p q^{2} S^{2}\left(\beta-(\gamma+\delta) q^{2} S^{2}\right)}{\beta^{2}+(\gamma-\delta) \beta q^{2} S^{2}-2 \gamma^{2} q^{4} S^{4}}
$$

Individual payoffs are given by:

$$
\begin{aligned}
& \tilde{u}_{1,2}=\frac{\beta p^{2} q^{2} S^{2}\left(\beta-\gamma q^{2} S^{2}\right)^{2}}{2\left(\beta^{2}+(\gamma-\delta) \beta q^{2} S^{2}-2 \gamma^{2} q^{4} S^{4}\right)^{2}} \\
& \tilde{u}_{3}=\frac{\beta p^{2} q^{2} S^{2}\left(\beta-(\gamma+\delta) q^{2} S^{2}\right)^{2}}{2\left(\beta^{2}+(\gamma-\delta) \beta q^{2} S^{2}-2 \gamma^{2} q^{4} S^{4}\right)^{2}}
\end{aligned}
$$

Notice that:

$$
\tilde{u}_{3}=\tilde{u}_{1,2} \frac{\left(\beta-(\gamma+\delta) q^{2} S^{2}\right)^{2}}{\left(\beta-\gamma q^{2} S^{2}\right)^{2}}<\tilde{u}_{1,2}
$$


which shows that, as expected, the unconnected agent enjoys lower profits in equilibrium compared to the connected agents, while aggregate profits are:

$$
\tilde{u}_{T}=\frac{\beta p^{2} q^{2} S^{2}\left(2\left(\beta-\gamma q^{2} S^{2}\right)^{2}+\left(\beta-(\gamma+\delta) q^{2} S^{2}\right)^{2}\right)}{2\left(\beta^{2}+(\gamma-\delta) \beta q^{2} S^{2}-2 \gamma^{2} q^{4} S^{4}\right)^{2}}
$$

For appropriators 1 and 2, individual profits in the fully connected network (b) are higher than those in the partly connected network (c), which in turn, are higher than the ones associated with the unconnected network (a), i.e., $\hat{u}_{1,2}>\tilde{u}_{1,2}>u_{1,2}^{*}$. What is interesting here is the case of the third appropriator. While the full connected network structure gives her the highest profits, $\left(\hat{u}_{3}>\tilde{u}_{3}\right.$ and $\left.\hat{u}_{3}>u_{3}^{*}\right)$, the comparison between profits in the partly connected network and profits in the unconnected network leads to a more surprising result. More precisely, for the "independent" appropriator, profits in the unconnected network are unambiguously higher than profits in the partly connected network (i.e., $u_{3}^{*}>\tilde{u}_{3}$ ). This is explained by the fact that the first two agents enjoy higher profits resulting from higher harvesting when they connect, which puts the last agent in a disadvantageous position. This leads to the following result.

Proposition 3 (Intensity of local interactions) Assume a partly connected network, where $g_{i j}=1$, for some pairs of agents and $g_{i j}=0$ for some other pairs. Then, stronger local interactions (i.e., higher $\delta$ value), will increase the individual profits of connected agents (say $i), \partial \tilde{u}_{i} / \partial \delta>0$, while the opposite is true for the individual profits of unconnected agents (sayj), $\partial \tilde{u}_{j} / \partial \delta<0$.

Proof The proof is available in "Appendix A.4" for an n-agent network.

This is an interesting result that analyses the relationship between network structure (the presence or absence of collaboration links between the agents) and the intensity of local interactions. It says that in the case of a partly connected network, an increase in the intensity of local interactions affects connected and unconnected agents differently. The former enjoy higher profits, while the latter have lower profits. This is, to the best of our knowledge, a new theoretical result that needs to be tested in different resource communities. In network games without global interactions effects, it has been shown that an increase in the intensity of social interactions increases individual profits of all the agents in the network (see for example, Helsley and Zenou 2014; Verdier and Zenou 2017). The presence of congestion externalities in our model, that characterizes the use of natural resources, increases the gap between the profits of connected and unconnected agents when $\delta$ increases. In fact, when $\delta$ increases, the harvesting cost of connected agents decreases while the cost of unconnected agents is relatively higher which leads to lower profits.

\section{Optimal Cake-Eating in the Network}

\subsection{Conservation and Optimal Use of the Resource}

When the global interaction effect is larger than the local interaction effect, the negative congestion externality dominates and there is too much extraction at the Nash 
equilibrium compared to the social optimum outcome, since individuals ignore the negative externality that their effort has on others. Also, the appropriators do not consider the scarce nature of the resource which leads to even further increases in the gap between the market and the optimal harvest. Then, there is room for government intervention which can take the form of a Pigouvian tax.

The regulator's objective is to maximize the sum of individual payoffs and at the same time consider the welfare loss that is associated with the reduction in the stock of the resource. If $\kappa>0$ denotes the (per unit) value of the unharvested resource then the social planner chooses $H_{1}, H_{2}, \ldots, H_{n}$ to maximize total welfare:

$$
\max _{\mathbf{H}^{o}} W^{o}\left(\mathbf{H}^{o}, \mathbf{g}\right)=\max _{H_{i}} \sum_{i=1}^{n} u_{i}\left(H_{i}^{o}, g\right)+\kappa\left(S-\sum_{i=1}^{n} H_{i}^{o}\right)
$$

The first-order necessary conditions for an interior solution are:

$$
\begin{aligned}
p-\kappa-\frac{\beta}{q^{2} S^{2}} H_{i}+2 \gamma H_{i}-2 \gamma \sum_{j=1}^{n} H_{j}+2 \delta \sum_{j=1}^{n} g_{i j} H_{j}=0 \\
H_{i}^{o}=\frac{p-\kappa-2 \gamma \sum_{j=1}^{n} H_{j}^{o}+2 \delta \sum_{j=1}^{n} g_{i j} H_{j}^{o}}{\frac{\beta}{q^{2} S^{2}}-2 \gamma}
\end{aligned}
$$

It is clear that for $\gamma>\delta, H_{i}^{o}<H_{i}^{*}$, i.e., individuals are harvesting too much at the Nash equilibrium as compared to the social optimum outcome.

In matrix form,

$$
\mathbf{H}^{o}(\boldsymbol{\Sigma})=\frac{p-\kappa}{\frac{\beta-2 \gamma q^{2} S^{2}}{q^{2} S^{2}}+2 \gamma b\left(\mathbf{g}, \frac{2 \delta q^{2} S^{2}}{\beta-2 \gamma q^{2} S^{2}}\right)} \mathbf{b}\left(\mathbf{g}, \frac{2 \delta q^{2} S^{2}}{\beta-2 \gamma q^{2} S^{2}}\right)
$$

The higher the interest of the regulator with respect to the conservation of the resource $(\kappa>0)$, the lower the optimal individual (and aggregate) harvesting amount, since $\frac{\partial H_{i}^{o}}{\partial \kappa}=-\frac{q^{2} S^{2}}{\beta-2 \gamma q^{2} S^{2}}<0$. This captures the fact that society has a benefit from preserving part of the natural resource for future use, which implies lower harvesting at the optimum. Another interesting observation concerns the "stock effect": $\frac{\partial H_{i}^{D}}{\partial S}>\frac{\partial H_{i}^{o}}{\partial S}>0$, where $H_{i}^{D}$ and $H_{i}^{o}$ are the optimal harvesting amounts when $\kappa=0$ and $\kappa>0$, respectively. This shows that increases in the stock of the resource will increase $H_{i}^{D}$ to a larger extent compared to the "optimal harvesting under preservation", $H_{i}^{o}$. In the second case, the trade-off between the benefit of harvesting a higher amount of the resource versus the benefit of preserving part of it leads to a lower resource exploitation. In other words, when the benefits of preserving part of the resource are taken into account by the social planner, the optimal harvesting amount is shown to be lower.

Note that the solution of the optimal cake-eating (28) is conditional on the existing network structure. In other words, in the maximization problem above the regulator does not choose the density of the network since the network structure is taken as given. Thus, the question remains: is there any socially optimal network structure? To answer this question, the social planner should determine, in addition to the optimal harvesting, an optimal link configuration, such as:

$$
\left\{g_{i j}^{*}\right\}, i, j=1, \ldots, n, g_{i i}=0 .
$$


Thus, the full social optimum, including the optimal network structure, will be determined by the solution to the problem:

$$
\begin{aligned}
\max _{\mathbf{H}, \mathbf{g}} W^{o}(\mathbf{H}, \mathbf{g}) & =\max _{H_{i}, g_{i j}} \sum_{i=1}^{n} u_{i}\left(H_{i}, g_{i j}\right)+\kappa\left(S-\sum_{i=1}^{n} H_{i}\right) \\
\text { subject to } \quad g_{i i} & =0, \quad g_{i j} \in\{0,1\}, \quad i, j=1, \ldots, n, \quad i \neq j .
\end{aligned}
$$

This is a concave mixed-integer, nonlinear optimization problem (MI-NLOP) and its solutions can be obtained using numerical optimization methods. In Sect. 7 below, we solve this problem numerically and derive both the optimal harvesting amount and the optimal network structure.

\subsection{Optimal Policy}

As discussed above, the optimal policy, in the case where the congestion effect dominates the positive externality, will take the form of a Pigouvian tax. This tax will depend explicitly on the network centrality of the agent and will internalize the net congestion externality that an agent imposes on the rest of the agents when harvesting a unit of the resource. In other words, an agent who is much more engaged in coordination/collaboration efforts trying to reduce the negative congestion effect will have to pay a lower amount in the form of taxation than an agent who acts more "independently".

If $\tau_{i}^{o}(\mathbf{g})$ denotes the optimal tax per unit of harvest, it is clear from the comparison between the equilibrium and the optimal FOC that the optimal tax is given by:

$$
\tau_{i}^{o}(\mathbf{g})=\gamma \sum_{j \neq i}^{n} H_{j}^{o}-\delta \sum_{j=1}^{n} g_{i j} H_{j}^{o}+\kappa
$$

for $\kappa \geq 0$. More central agents are connected to a higher number of appropriators, which increases the term $\left(\delta \sum_{j=1}^{n} g_{i j} H_{j}^{o}\right)$ in the RHS of Eq. (30). This implies a lower agent-specific tax as compared to the tax imposed on a "less-connected" agent.

The timing will be as follows: first, the government will announce a tax per unit of resource extracted that will be equal to $\tau_{i}^{o}(\mathbf{g}) \geq \mathbf{0}$, for each individual $i=1, \ldots, n$. Then $i$ will choose the amount of the resource use that will maximize her payoff:

$$
u_{i}=\left(p-\tau_{i}^{o}\right) H_{i}-\frac{1}{2}\left(\frac{\beta}{q^{2} S^{2}}\right) H_{i}^{2}-\gamma \sum_{j \neq i}^{n} H_{i} H_{j}+\delta \sum_{j=1}^{n} g_{i j} H_{i} H_{j}
$$

This allows the social planner to restore the optimum as an equilibrium outcome and to "punish" collaborative agents relatively less. The optimal policy is stricter in case the regulator follows a conservation plan that takes into account the value of the unharvested resource $(\kappa>0)$.

To determine the tax and express it in terms of Bonacich centrality, we follow the process below. When the regulator imposes a tax $\tau_{i}$ per unit $H_{i}$, the agents take the tax rate as given and maximize their profits. Then, the Nash equilibrium $\left(\mathbf{H}_{\tau}^{*}\right)$ under a non-uniform tax should solve: 


$$
-\boldsymbol{\Sigma} \cdot \mathbf{H}_{\tau}^{*}=\left[\frac{\beta-\gamma q^{2} S^{2}}{q^{2} S^{2}} \mathbf{I}+\gamma \mathbf{U}-\delta \mathbf{G}\right] \cdot \mathbf{H}_{\tau}^{*}=p-\boldsymbol{\tau}
$$

where $\boldsymbol{\tau}$ is the vector of agent-specific taxes, $\boldsymbol{\tau}=\left(\tau_{1}, \ldots \tau_{n}\right)$.

In "Appendix A.5" we show that the harvesting amount is given by:

$$
\mathbf{H}_{\tau}^{*}(\boldsymbol{\Sigma})=\frac{1}{\frac{\beta-\gamma q^{2} S^{2}}{q^{2} S^{2}}+\gamma b\left(\mathbf{g}, \frac{\delta q^{2} S^{2}}{\beta-\gamma q^{2} S^{2}}\right)}\left[p \mathbf{b}\left(\mathbf{g}, \frac{\delta q^{2} S^{2}}{\beta-\gamma q^{2} S^{2}}\right)-\mathbf{b}_{\tau}\left(\mathbf{g}, \frac{\delta q^{2} S^{2}}{\beta-\gamma q^{2} S^{2}}\right)\right]
$$

where $\mathbf{b}_{\tau}\left(\mathbf{g}, \frac{\delta q^{2} S^{2}}{\beta-\gamma q^{2} S^{2}}\right)=\mathbf{M}\left(\mathbf{g}, \frac{\delta q^{2} S^{2}}{\beta-\gamma q^{2} S^{2}}\right) \cdot \boldsymbol{\tau}$ is the weighted Bonacich centrality, and the vector of the optimal tax is: ${ }^{11}$

$$
\boldsymbol{\tau}=\left[\frac{\beta-\gamma q^{2} S^{2}}{q^{2} S^{2}}+\gamma b\left(\mathbf{g}, \frac{\delta q^{2} S^{2}}{\beta-\gamma q^{2} S^{2}}\right)\right]\left[\mathbf{M}\left(\mathbf{g}, \frac{\delta q^{2} S^{2}}{\beta-\gamma q^{2} S^{2}}\right)\right]^{-1}\left(\mathbf{H}^{*}-\mathbf{H}^{o}\right)
$$

It is clear from both (30) and (34) that the optimal policy is an agent-specific tax, which depends on the centrality of each agent. This leads to the following proposition:

Proposition 4 (Optimal level of taxation) Assuming that $\delta \lambda_{1}(\mathbf{g})<\frac{\beta}{q^{2} S^{2}}-\gamma$, then a tax of the form $\tau_{i}^{o}(\mathbf{g})=\gamma \sum_{j \neq i}^{n} H_{j}^{o}-\delta \sum_{j=1}^{n} g_{i j} H_{j}^{o}+\kappa$ will implement the optimal allocation as an equilibrium outcome. The social planner will impose lower taxes on more central agents in the network.

Proof When the regulator announces a tax per unit of resource extracted which is equal to $\tau_{i}^{o}(\mathbf{g})$, then agents maximize their payoffs given by (31). It is easy to see that the firstorder condition derived by (31) is the same as the optimal FOC given by (27). That is, the equilibrium harvesting amount when an agent-specific tax is enforced, $\tau_{i}^{o}(\mathbf{g})$, is equal to the optimal harvesting amount.

Proposition 5 (Taxes and network structure) (1) Partly Connected Network: The optimal harvesting as an equilibrium outcome can only be achieved by using the agent-specific taxation described in Proposition 3. Uniform taxes do not lead to the optimal amount of harvesting. (2) Full or Unconnected Network and Symmetric Agents: The tax is, however, uniform when all the agents are connected to each other or when there are no collaboration links between the resource users.

Proof (1) Agents can be heterogeneous with respect to the number of links they form with their co-appropriators, which implies that the individual net congestion externality is different for different agents. However, the optimal taxation should fully internalize this externality, which means that different levels of the associated external effect lead to different tax levels. Also, the derived equilibrium level of resource exploitation is unique, and any other level of taxation will not satisfy the zero profit condition for the same amount of the resource harvested, and will not constitute an equilibrium outcome. (2) In the particular case of symmetric agents who are all connected to each other, the optimal tax, as it is given by (30), is uniform. Symmetry $\left(H^{o}=H_{j}^{o}, j=1, \ldots n\right)$ and full network of $n$ agents implies

$\overline{11}$ This is in line with Remark 1 of Ballester et al. (2006) which explains the case of heterogeneous agents. 
that the optimal tax becomes $\hat{\tau}^{o}=(\gamma-\delta)(n-1) H^{o}+\kappa$, which is lower than the optimal tax of the unconnected network $\left(\tau^{o *}=\gamma(n-1) H^{o}+\kappa\right)$.

Note that the optimal policy, as described above, will restore the first best in a given network structure. However, it will not induce the optimal network structure. If the existing stable market structure for the network is different from the optimal structure then the regulator will have to implement the optimal structure with other instruments (e.g., command and control).

An alternative policy instrument that is proposed in similar contexts is the use of permits. In terms of our model, if permits are to be used then the number of permits should restrict the use of the resource and the optimal number of permits should be equal to $\mathbf{H}^{o}$. In other words, the number of permits will be equal to the total amount of harvesting at the optimum. However, permits trading (if allowed) would result in an equilibrium price level which would be the same for all the agents. On the contrary, in the case of taxation, appropriators pay an agent-specific per-unit tax, which, as has been extensively explained above, differs with respect to their centrality in the network. Thus, it is clear that tradable permits can only be used as a second-best instrument.

\subsection{Example}

\subsubsection{A 3-Agent Star Network}

The difference between the market and the optimal outcomes can be illustrated in the small numbers network. Let us use the 3-agent example that was presented above (Sect. 3.2) and derive the optimum in the presence of the global interaction effect. In this case, the regulator will choose $H_{1}, H_{2}, H_{3}$, to maximize total welfare. Then, the optimal use of the resource is given by:

$$
H_{i}^{S}=\frac{p q^{2} S^{2}}{\beta+4 \gamma q^{2} S^{2}},
$$

and the optimal level of aggregate profits:

$$
W\left(H_{i}^{S}\right)=\frac{6 \gamma p^{2} q^{4} S^{4}}{\left(\beta+4 \gamma q^{2} S^{2}\right)^{2}}
$$

Comparing the market (11) and the optimal harvesting (35) in a network where there is only one negative externality, namely congestion, it is easy to see that harvesting is lower at the optimum. Agents should reduce the amount of the resource they are exploiting in order to reduce the negative effects that are imposed on the rest of the agents. A tax per unit of output that will fully internalize the damage caused by congestion will increase the cost of harvesting and will reduce the use of the resource to the optimal level. 


\subsubsection{A 3-Agent Full Network}

Let us now turn to the case where all externalities are present and agents have created collaboration links between each other. The regulator takes into account these positive local interactions and the optimal use of the resource is given by:

$$
\hat{H}_{i}^{S}=\frac{p q^{2} S^{2}}{\beta+4(\gamma-\delta) q^{2} S^{2}}
$$

while the optimal value of the network is given by:

$$
W\left(\hat{H}_{i}^{S}\right)=\frac{6(\gamma-\delta) p^{2} q^{4} S^{4}}{\left(\beta+4(\gamma-\delta) q^{2} S^{2}\right)^{2}}
$$

It is interesting to again compare the optimal use of the resource, with the decentralized use in the corresponding case, $\hat{H}_{i}=\frac{p q^{2} S^{2}}{\beta+2(\gamma-\delta) q^{2} S^{2}}$. If the global interaction effect, $\gamma$, is higher than the local interaction effect, $\delta$, the use of the resource will be smaller at the optimum than in the decentralized case, $\hat{H}_{i}^{S}<\hat{H}_{i}$. Internalizing the net congestion effect leads to lower use of the resource at the optimum. Notice that the optimal value of the network (or else aggregate welfare) under the presence of two externalities (given by 38) is higher than the optimal value of the network under congestion externality (described by 36) which clearly shows that the regulator has an incentive to enforce policies that will promote the collaboration between the agents.

\subsubsection{Optimal Taxation}

We can now calculate the optimal taxation for all the network structures presented in Fig. 2. This tax will restore the optimum for a given network structure. In the case of symmetric agents, in a star network (Fig. 2a), the optimal tax is the same for all individuals and is equal to:

$$
t_{S}=\frac{2 \gamma p q^{2} S^{2}}{\beta+4 \gamma q^{2} S^{2}}
$$

while in the full network (Fig. 2b), the optimal tax is clearly lower than the one imposed on the appropriators of the star network, or:

$$
t_{F}=\frac{2(\gamma-\delta) p q^{2} S^{2}}{\beta+4(\gamma-\delta) q^{2} S^{2}}<t_{S}
$$

In the partly connected network (Fig. 2c), the tax imposed on the connected agents ( 1 and 2 ) is different than the one imposed on the unconnected agent. In particular, the connected agents have to pay a per unit of harvest tax, equal to:

$$
t^{1,2}=\frac{p q^{2} S^{2}\left[\beta(2 \gamma-\delta)-4 \gamma^{2} q^{2} S^{2}\right]}{\Omega}
$$

where $\Omega=\beta^{2}+2(\gamma-\delta) \beta q^{2} S^{2}-8 \gamma^{2} q^{4} S^{4}$, while the unconnected agent will pay a higher tax given by: 


$$
t^{3}=\frac{2 p q^{2} S^{2} \gamma\left(\beta-2 q^{2} S^{2} \gamma\right)}{\Omega}>t^{1,2}
$$

The higher tax punishes agent 3 in the partly connected network for the externality she imposes on the rest of the agents, without making any effort to reduce the magnitude of the externality (since $g_{i 3}=0$,for $i=1,2$ ). In other words, agent 3 now has a stronger incentive to create a link with the other two appropriators since non-collaboration implies a higher cost. The comparison of agent-specific taxes in the three network structures gives: $t_{S}>t^{1,2}>t_{F}$ for agents 1 and 2 , and $t^{3}>t_{S}>t_{F}$ for agent 3 . It is clear that it is more costly for agent 3 to stay unconnected in the partly connected network as compared to the star network.

\section{Dynamics of Network Formation}

In this section we examine whether the appropriators in our resource network will decide to create or sever links. We develop a simplified framework where at the beginning of the period the agents, given full information about congestion costs and cooperation spillovers, are going to decide to create new links or sever existing cooperation links. Their decisions will determine the structure of the network at the end of the period. ${ }^{12}$

The same approach can be applied to determine the socially efficient network structure. The regulator decides at the beginning of the period to retain or sever cooperation links using as an objective the maximization of aggregate payoffs plus the conservation value of the resource. The structure of the network at the end of the period will characterize the socially efficient network structure. If this structure is different from the efficient "market structure" the regulator has an incentive to intervene in order to provide incentive schemes which will attain the socially efficient market structure.

\subsection{Market Network Structure}

Assume that at the beginning of the period the network is star-shaped, that is, no cooperation links exist. For simplicity, we will use the 3-agent example presented above, but our results can be generalized in an $n$-agent network. Let $u_{i}^{*}\left(\mathbf{H}^{S} \mid-i j\right), i, j=1,2,3, i \neq j$, denote the maximized payoff of each agent, given that no links exist, with $\mathbf{H}^{S}$ the vector of profit-maximizing harvesting when the network is star-shaped. For cooperation to emerge it should be profitable for both agents. Then, the following results can be stated, where $\mathbf{H}$ denotes the vector of profit-maximizing harvesting at each network structure, $+i j$ means that the link $i j$ is created and $-i j$ means that the link is not created, for $i, j=1,2,3, i \neq j$.

\footnotetext{
12 It should be noted that this not a fully dynamic set-up. A fully dynamic set-up would consider a multiperiod problem and examine the convergence to a stable network structure. Such a problem has been studied by Watts (2001) and Jackson and Watts (2002). In these papers, the payoff functions were, however, relatively simple and allowed analytic results. We regard our approach to the problem as a first-order approach with the development of a fully dynamic model being the next stage of our research.
} 
a If

$$
\begin{aligned}
& u_{1}(\mathbf{H} \mid+12,-13)>u_{1}^{*}\left(\mathbf{H}^{S} \mid-i j\right) \text { and } u_{1}(\mathbf{H} \mid+12,-13)>u_{1}(\mathbf{H} \mid+i j), \\
& u_{2}(\mathbf{H} \mid+21,-23)>u_{2}^{*}\left(\mathbf{H}^{S} \mid-i j\right) \text { and } u_{2}(\mathbf{H} \mid+21,-23)>u_{2}(\mathbf{H} \mid+i j),
\end{aligned}
$$

then, there will be a link between 1 and 2 but not with 3 .

Note that it is possible to have:

$$
\begin{aligned}
u_{3}(\mathbf{H} \mid+i j) & >u_{3}^{*}\left(\mathbf{H}^{S} \mid-i j\right) \\
\text { or, } & u_{3}(\mathbf{H} \mid+13,-23)>u_{3}^{*}\left(\mathbf{H}^{S} \mid-i j\right) \\
\text { or, } & u_{3}(\mathbf{H} \mid+23,-13)>u_{3}^{*}\left(\mathbf{H}^{S} \mid-i j\right)
\end{aligned}
$$

but there will be no cooperation with 3 since this is not profitable for the other agents. Any equilibrium should satisfy the pairwise stability condition, which accounts for the mutual approval of both agents. Similar inequalities hold in the case where any two agents find it profitable to connect without including the third one.

Notice that if

$$
\begin{aligned}
& {\left[u_{3}(\mathbf{H} \mid+13,-23)-u_{3}^{*}\left(\mathbf{H}^{S} \mid-i j\right)\right]>\left[u_{1}(\mathbf{H} \mid+12,-13)-u_{1}(\mathbf{H} \mid+i j)\right]} \\
& {\left[u_{3}(\mathbf{H} \mid+23,-13)-u_{3}^{*}\left(\mathbf{H}^{S} \mid-i j\right)\right]>\left[u_{2}(\mathbf{H} \mid+21,-23)-u_{2}(\mathbf{H} \mid+i j)\right]}
\end{aligned}
$$

agent 3 could bribe agents 1 and 2 in order to convince them to cooperate with her. $\mathrm{b}$ If

$$
u_{i}(\mathbf{H} \mid+i j)>u_{i}^{*}\left(\mathbf{H}^{S} \mid-i j\right), i, j=1,2,3, i \neq j,
$$

then, all agents create cooperation links.

c If

$$
u_{i}(\mathbf{H} \mid+i j)<u_{i}^{*}\left(\mathbf{H}^{S} \mid-i j\right), i, j=1,2,3, i \neq j,
$$

no cooperation links are created and the star-shaped network will remain until the end of the period.

The networks created in cases (a)-(c) are pairwise stable in the sense of Jackson and Wolinsky (1996). The efficiency of the network depends on the maximization of the individual payoffs:

$$
u_{h}^{M}=\sum_{i=1}^{3} u_{i h}, h=a, b, c
$$

The strongly efficient network is the one that implies the maximum aggregate payoffs, or:

$$
u_{h}^{M}=\arg \max _{h} \sum_{i=1}^{3} u_{i h}, h=a, b, c
$$

In our resource problem with homogeneous agents, denser networks always increase aggregate harvesting and payoffs (see Proposition 2), since they decrease the magnitude of the congestion externality. As a result, the strongly efficient network is always the fully connected one. This is in line with the empirical literature where it has been shown that natural resource management is more effective and efficient if resource users participate in 
planning processes and communicate with their peers (see e.g. Layzer 2008; Kubo and Supriyanto 2010; Ramirez-Sanchez 2011). Introducing heterogeneity though (see Sects. 6, 7) can lead to different outcomes.

\section{Extensions: Introducing Heterogeneity}

Our theoretical model in Sect. 3 can provide useful insights on how collective action can lead to more efficient use of natural resources. To derive our results we assumed that individual agents are homogeneous. However, it is important to bear in mind that resource communities are rarely one coherent group of appropriators; rather, they are defined by subgroups with different perceptions, interests, extractions costs, and amount of influence (Agrawal and Gibson 1999; Carlsson and Berkes 2005; Nygren 2005; Crona and Bodin 2011). In this section, we will change this assumption, and we will show how heterogeneity, in terms of geographical distance to the resource and firms' size, affects the equilibrium harvesting amount and the network structure. In the next section we will use numerical examples to show how heterogeneity affects the incentives of individual agents to collaborate with the rest of the resource users.

\subsection{Costly Transportation}

Let us assume now that the transportation of the resource is costly and the cost depends on the geographical distance between the agent and the resource. Until now, we had assumed that the agents involved in the exploitation of the resource were homogeneous. Costly transportation introduces heterogeneity in the model, since agents who locate further away from the resource have to pay a higher transportation cost, which increases the total cost of harvesting, affects their harvesting decisions and decreases their payoffs.

Let $l_{i} \in(0,1]$ represent the location of the agent $i$, defined as her distance from the resource, and $\xi$ represents the marginal transportation cost.

Then, using the notation $\hat{p}_{i}=p-\xi l_{i}$, Eq. (7) becomes:

$$
-\boldsymbol{\Sigma} \cdot \mathbf{H}=\left[\frac{\beta-\gamma q^{2} S^{2}}{q^{2} S^{2}} \mathbf{I}+\gamma \mathbf{U}-\delta \mathbf{G}\right] \cdot \mathbf{H}=\hat{\mathbf{p}}
$$

or,

$$
\frac{\beta-\gamma q^{2} S^{2}}{q^{2} S^{2}}\left[\mathbf{I}-\frac{\delta q^{2} S^{2}}{\beta-\gamma q^{2} S^{2}} \mathbf{G}\right] \cdot \mathbf{H}^{*}=\hat{\mathbf{p}}-\gamma H^{*} \cdot \mathbf{1}
$$

By inverting the matrix (46) and doing some calculations that are described in "Appendix A.6", we get:

$$
\mathbf{H}^{*}(\boldsymbol{\Sigma})=\frac{1}{\frac{\beta-\gamma q^{2} S^{2}}{q^{2} S^{2}}+\gamma b\left(\mathbf{g}, \frac{\delta q^{2} S^{2}}{\beta-\gamma q^{2} S^{2}}\right)} \mathbf{b}_{\hat{\mathbf{p}}}\left(\mathbf{g}, \frac{\delta q^{2} S^{2}}{\beta-\gamma q^{2} S^{2}}\right)
$$

where $\mathbf{b}_{\hat{\mathbf{p}}}\left(\mathbf{g}, \frac{\delta q^{2} S^{2}}{\beta-\gamma q^{2} S^{2}}\right)$ is the weighted Bonacich centrality: 


$$
\begin{aligned}
& b_{\hat{p}_{i}}\left(\mathbf{g}, \frac{\delta q^{2} S^{2}}{\beta-\gamma q^{2} S^{2}}\right)=\left[\mathbf{I}-\frac{\delta q^{2} S^{2}}{\beta-\gamma q^{2} S^{2}} \mathbf{G}\right]^{-1}\left(p-\xi l_{i}\right) \\
& =p b_{i}\left(\mathbf{g}, \frac{\delta q^{2} S^{2}}{\beta-\gamma q^{2} S^{2}}\right)-\xi \mathbf{M}\left(\mathbf{g}, \frac{\delta q^{2} S^{2}}{\beta-\gamma q^{2} S^{2}}\right) \cdot \mathbf{l}
\end{aligned}
$$

and $\mathbf{I}$ is the vector of individual locations. The equilibrium condition (47) shows that higher transportation cost increases the cost of harvesting and leads to lower use of the resource. This implies that agents who are located further away find it profitable to harvest a smaller amount of the resource compared to those that are located closer to the resource.

It is interesting to explore whether transportation costs and heterogeneity will make the formation of links easier, meaning that the agents will have a higher incentive to collaborate with each other. In order to do so we need to compare individual payoffs in different cases. If $\bar{u}_{i}, u_{i}^{* *}$ denotes the payoff of agent $i$ in a fully connected and unconnected network, respectively, with heterogeneity then transportation costs make the formation of links easier iff:

$$
\bar{u}_{i}(\mathbf{H} \mid+i j)-u_{i}^{* *}\left(\mathbf{H}^{S} \mid-i j\right)>\hat{u}_{i}(\mathbf{H} \mid+i j)-u_{i}^{*}\left(\mathbf{H}^{S} \mid-i j\right)
$$

That is, heterogeneity creates a larger difference between the payoffs of the fully connected network $\left(\bar{u}_{i}\right)$ and the corresponding ones of the star network $\left(u_{i}^{* *}\right)$. In terms of the inequality above, the LHS shows the difference between the payoffs of the connected and the unconnected network in case of heterogeneity, while the RHS shows the same difference in case all agents locate at an equal distance from the resource (or more generally, when transportation is not costly or the per unit cost is not very high). Numerical simulations will give a clearer idea of whether heterogeneity could facilitate the creation of links between the agents.

Using the three agent example analyzed above, we can compare the case of costly transportation to one that involves no cost. In particular, the comparison between the payoffs of the connected network in both of these cases leads to the following condition:

$$
\begin{aligned}
\bar{u}_{i}(\mathbf{H} \mid+i j) & >\hat{u}_{i}(\mathbf{H} \mid+i j) \Rightarrow \\
\frac{l_{i}}{\sum_{j \neq i} l_{j}} & <\frac{\gamma-\delta}{\left(\frac{\beta-\gamma q^{2} S^{2}}{q^{2} S^{2}}+2 \gamma-\delta\right)} \Rightarrow \\
l_{i} & <\lambda \sum_{j \neq i} l_{j}
\end{aligned}
$$

where $\lambda=(\gamma-\delta) /\left(\frac{\beta-\gamma q^{2} S^{2}}{q^{2} S^{2}}+2 \gamma-\delta\right)$ and $0<\lambda<1$. Inequality (49) shows that costly transportation can, surprisingly, be more profitable for agent $i$ than non-costly transportation, iff the distance between agent $i$ and the resource is smaller than the sum of the distance between the rest of the agents and the resource, multiplied by a positive number $\lambda$. It is interesting to notice that $\lambda$ is increasing in $\gamma$ and decreasing in $\delta$. That is, it is easier for agent $i$ to obtain higher profits under costly transportation when the negative congestion effect is stronger or when she enjoys lower benefits from collaborating with the rest of the agents. In general, costly transportation can be shown to be beneficial for the agent who is located closer to the resource. Note that the per unit transportation cost, $\xi$, does not affect this result. The only thing that matters is how much "closer" to the resource agent $i$ is located compared to the co-appropriators. 
Table 1 Bonacich measure $\left(\mathrm{b}_{\mathrm{i}}\right)$, individual harvesting amount $\left(\mathrm{H}_{\mathrm{i}}\right)$ and individual profits $\left(\mathrm{u}_{\mathrm{i}}\right)$ for the four network structures of (Figure 3)

\begin{tabular}{lllllll}
\hline Agent type & $b_{i}$ & $H_{i}$ & $u_{i}$ & $b_{i}$ & $H_{i}$ & $u_{i}$ \\
\hline & $($ a) & & & (b) & & \\
1 & 1 & 0.30364 & 0.22128 & 1.0021 & 0.30427 & 0.22220 \\
2 & 1 & 0.30364 & 0.22128 & 1 & 0.30363 & 0.22127 \\
& $($ c) & & & (d) Equilibrium & & \\
1 & 1.0146 & 0.30806 & $\mathbf{0 . 2 2 7 7 7}$ & 1.01486 & 0.30801 & 0.22768 \\
2 & 1.0021 & 0.30425 & 0.22216 & 1.01486 & 0.30801 & $\mathbf{0 . 2 2 7 6 8}$ \\
\hline
\end{tabular}

\subsection{The Size of Firms}

A second interesting case that introduces heterogeneity in the model is the case where the agents, or the exploiting firms, are of different size. Let us assume here that there is one big firm, the "leader" $(L)$ and a number of smaller ones $(F)$ of equal size, the "followers." In terms of our modeling, this will lead to different private costs. In particular, the marginal cost of the larger firm will be lower than the corresponding cost of the smaller firms, i.e., $\beta_{L}<\beta_{F}$.

If such an equilibrium exists, then it solves: ${ }^{13}$

$$
-\boldsymbol{\Sigma} \cdot \mathbf{H}=\left[\left(\frac{1}{q^{2} S^{2}}\right) \mathbf{B}-\gamma \mathbf{I}+\gamma \mathbf{U}-\delta \mathbf{G}\right] \cdot \mathbf{H}=p \cdot \mathbf{1} .
$$

where

$$
\mathbf{B}=\left(\begin{array}{ccc}
\beta_{L} & \cdots & 0 \\
\vdots & \ddots & \vdots \\
0 & \cdots & \beta_{F}
\end{array}\right)
$$

By using simple algebra, the equilibrium resource use is given by: ${ }^{14}$

$$
\mathbf{H}^{*}=p \Phi^{-1} \mathbf{b}\left(\mathbf{g}, \frac{\delta q^{2} S^{2}}{1-\gamma q^{2} S^{2}}\right)
$$

where $\Phi$ denotes the matrix: $\Phi=\left[\left(\frac{1}{q^{2} S^{2}}\right) \mathbf{M}^{-1} \mathbf{X}+\left(\left(\frac{1}{q^{2} S^{2}}-\gamma\right)+\gamma H^{*} b\right) \mathbf{I}\right]$.

The numerical exercise of Sect. 7 will provide interesting insights into the incentives of the two types of agents to connect or not. More specifically, we will see that if the leader has a large competitive advantage, by being able to produce at a much lower cost (in which case $\beta_{L}$ is significantly lower than $\beta_{F}$ ), and uses advance technology, meaning that smaller firms would benefit more from collaborating with the leader rather than the opposite $\left(\delta_{L}<\delta_{F}\right)$, then there is no incentive for the large firm to collaborate with the competitors.

13 Here the condition of existence becomes: $\frac{\beta_{L}}{q^{2} S^{2}}-\gamma>\delta \lambda_{1}(\mathbf{G})$.
14 The steps are available in "Appendix A.7" 


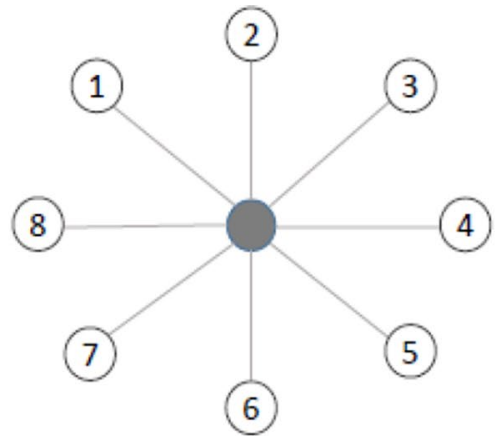

(a)

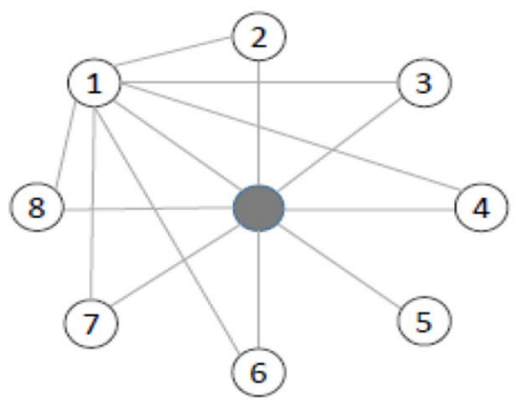

(c)

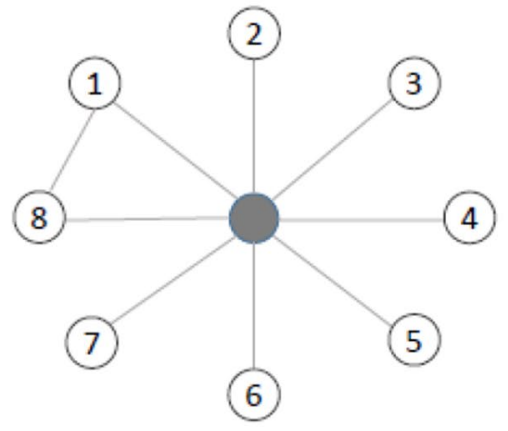

(b)

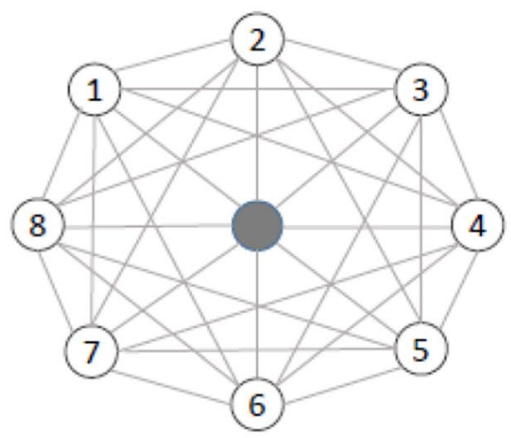

(d) Equilibrium

Fig. 3 Symmetric agents: parameter values: $\mathrm{S}=5, \mathrm{q}=0.1, \beta=1.2, \delta=0.01, \gamma=0.02, p=1.5$

In this case, the leading firm will keep exploiting a large part of the resource without being willing to share any knowledge with the followers.

If the regulator wants to protect the smaller appropriators and works toward the formation of a fully connected network, she will have to impose a stricter regulation to the large firm, in the form of taxation, which will fully internalize the negative congestion effects that she imposes on the rest of the appropriators. This is in line with our policy analysis above.

\section{Numerical Example}

\subsection{Symmetric Agents}

Let us provide some more insightful examples by using an 8-agent network and solve for the market and the optimal solution numerically. In Fig. 3, we consider four different network structures: the star network (a), the case where there is only one collaboration link 
Table 2 Individual harvesting amount $\left(\mathrm{H}_{\mathrm{i}}\right)$ and individual profits $\left(\mathrm{u}_{\mathrm{i}}\right)$ for the three network structures of (Figure 4)

\begin{tabular}{lllllll}
\hline Agent Type & $H_{i}$ & $u_{i}$ & $H_{i}$ & $u_{i}$ & $H_{i}$ & $u_{i}$ \\
\hline & (a) & & (b) Equilibrium & & (c) & \\
1 & 0.2999 & 0.2159 & 0.3036 & 0.2213 & 0.3061 & $\mathbf{0 . 2 2 4 9}$ \\
2 & 1.2150 & $\mathbf{0 . 8 8 5 7}$ & 1.2145 & 0.8851 & 1.2144 & 0.8849 \\
\hline
\end{tabular}

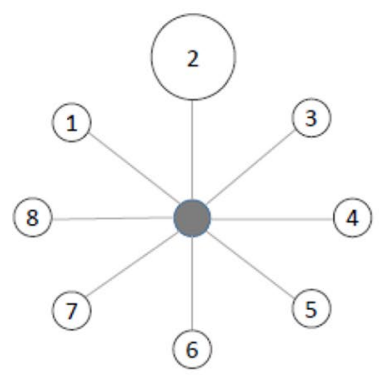

(a)

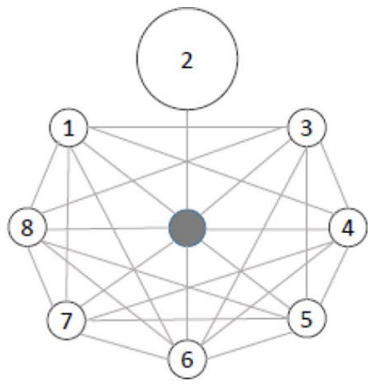

(b) Equilibrium

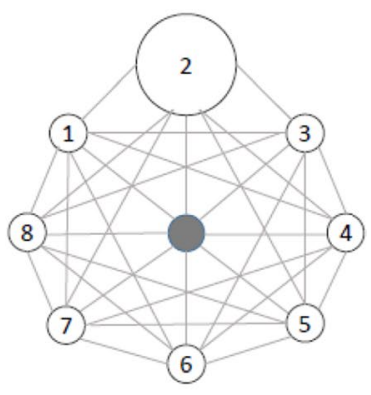

(c)

Fig. 4 Asymmetric agents: firms of different size. Parameter values: $\mathrm{S}=5, \mathrm{q}=0.1, \beta_{\mathrm{i}}=1.2, \beta_{2}=0.3, \delta_{i}=$ $0.01, \delta_{2}=0.0001, \gamma=0.02, p=1.5, \mathrm{i}=1,3, \ldots, 8$

between two agents (b), the case where one agent is connected to the rest of the agents (c), and the full network (d). Table 1 gives the Bonacich measure, the individual harvesting amount and the corresponding individual profits for the four network structures shown in Fig. 3. In Table 1, Agent Type 1 refers to agent 1 in Fig. 3, while Agent Type 2 refers to the rest of the agents. It is easy to observe that all the agents enjoy the highest profits in the full network, apart from agent 1 whose profits are higher in network (c). This network, though, is not pairwise stable, since all agents will find it profitable to connect with each other which will lead to network (d), which is the pairwise stable equilibrium of this game.

By solving the full optimal problem given by (29) numerically, where the planner will determine both the optimal harvesting amount and the optimal link configuration, we find that the optimal network structure is the equilibrium one, or else the full network. This is the case for any value of $\kappa \geq 0$. When the equilibrium network structure coincides with the optimal one, the planner only needs to control for the level of individual harvest by implementing the optimal agent-specific tax. In this numerical example, the optimal, individual harvesting amount is 0.30364 (or 0.28340 in conservation, with $\kappa=0.1)<H_{i}^{(d)}$.

\subsection{Asymmetric Agents}

\subsubsection{The Size of the Firms}

Not surprisingly, in the case of symmetric agents, the full network is shown to be the pairwise stable equilibrium, as well as the optimal network configuration. We will now show that introducing asymmetry in the game could lead to different results, where the market structure is different from the optimal one. In such a case, as explained above, the planner, 
Table 3 Individual harvesting amount $\left(\mathrm{H}_{\mathrm{i}}\right)$ and individual profits $\left(\mathrm{u}_{\mathrm{i}}\right)$ for the three network structures of (Figure 5)

\begin{tabular}{lllllll}
\hline Agent Type & $H_{i}$ & $u_{i}$ & $H_{i}$ & $u_{i}$ & $H_{i}$ & $u_{i}$ \\
\hline & (a) & & (b) & & (c) Equilibrium & \\
1 & 0.2002 & 0.0962 & 0.2001 & 0.0961 & 0.2042 & $\mathbf{0 . 1 0 0 1}$ \\
2 & 0.2421 & 0.1406 & 0.2419 & 0.1405 & 0.2459 & $\mathbf{0 . 1 4 5 2}$ \\
3 & 0.2839 & 0.1876 & 0.2868 & 0.1974 & 0.2877 & $\mathbf{0 . 1 9 8 6}$ \\
\hline
\end{tabular}

along with the agent-specific taxation that aims to restrict individual harvesting, should use some other policy instrument to create or sever collaboration links between the agents.

Figure 4 illustrates the case where there is a big player in the market (agent 2) and seven smaller resource users. The leading firm has a cost advantage, and thus her private cost of harvesting is lower than the corresponding one of the rest of the agents, $\beta_{2}<\beta_{i}$, $i=1,3, \ldots 8$. Also, the benefit of the larger player when collaborating with the rest of the agents is lower, indicating the fact that she already uses some more advance technology in the harvesting of the resource and thus the exchange of knowledge benefits more firms of smaller size $\left(\delta_{2}<\delta_{i}, i=1,3, \ldots 8\right)$.

The results in this case are interesting. As shown in Table 2, individual profits for smaller users (Agent Type 1) are higher in the case of the full network (c), followed by the corresponding ones in the partly connected network (where small users are connected only to each other without including the big player), while profits are lower in the star network (a). This is not the case, though, for the big firm. In particular, the big player enjoys the highest profits in the star network. It is not profitable for her to connect to any smaller user, while the highest profits for her are associated with the network where there are no collaboration links between any agent, i.e., $u_{2}^{(a)}>u_{2}^{(b)}>u_{2}^{(c)}$. However, the star network violates the pairwise stability condition. This is why the profits of the rest of the agents are higher in the full network, followed by the partly-connected network, and then by the star network, i.e., $u_{i}^{(a)}<u_{i}^{(b)}<u_{i}^{(c)}$. So, although the big player will decide not to connect with the rest of the agents (which impedes the creation of a full network), the small players will still have an incentive to collaborate with each other. Thus, the pairwise stable equilibrium configuration is the partly connected network (b).

Deriving numerically the optimal network structure, we observe that the full network is the optimal one. In this case (with $\kappa=0$ ), the optimal harvesting amount of the small agents is equal to $0.2975<H_{i}^{(b)}$ and the corresponding one for the big agent is $1.198<H_{2}^{(b)}$. The planner will have to come up with policies that will incentivize the big player to create collaboration links with the smaller ones (such as a subsidy per new link created). The communication failure of heterogeneous agents is in line with the findings of the experimental literature (e.g. Hackett et al. 1994; Gangadharan et al. 2017; Lacomba et al. 2017), according to which it is more difficult for heterogeneous populations to reach efficient outcomes. More precisely, related work confirms our theoretical findings and suggests that actors occupying central positions between subgroups may impede collaboration because they may be uninterested in fostering joint action or unwilling to share their advanced knowledge or technology (Bodin and Crona 2009). Thus, identifying these actors, bringing them into the decision making process and encouraging their collaboration with the rest of the users is likely to benefit the long-term sustainability of the resource system, which can be classified as a 'network intervention' (Valente 2012). 


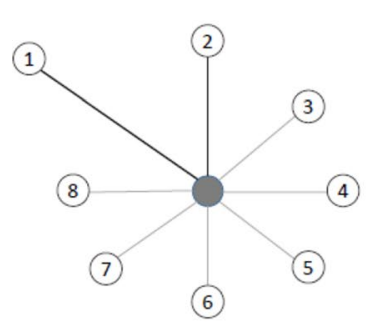

(a)

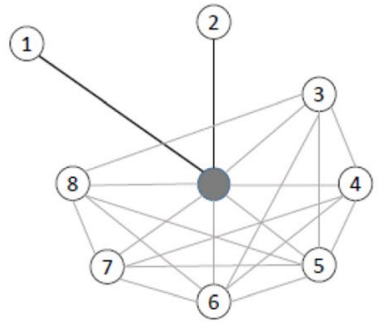

(b)

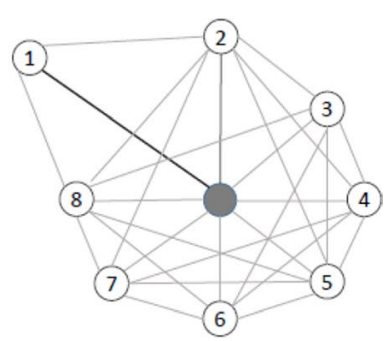

(c) Equilibrium

Fig. 5 Asymmetric agents: costly transportation. Parameter values: $S=5, q=0.1, \beta=1.2, \delta=0.01, \gamma=$ $0.02, p=1.5,1_{1}=0.5,1_{2}=0.3,1_{3}=0.1$

Table 4 Bonacich measure $\left(\mathrm{b}_{\mathrm{i}}\right)$, individual harvesting amount $\left(\mathrm{H}_{\mathrm{i}}\right)$ and individual profits $\left(\mathrm{u}_{\mathrm{i}}\right)$ for the two network structures of (Figure 6)

\begin{tabular}{lllllll}
\hline Agent Type & $b_{i}$ & $H_{i}$ & $u_{i}$ & $b_{i}$ & $H_{i}$ & $u_{i}$ \\
\hline
\end{tabular}

(a)

(b) Equilibrium

$\begin{array}{lllllll}1 & 1 & 0.3036 & 0.221 & 1.0042 & 0.3048 & \mathbf{0 . 2 2 3}\end{array}$

$\begin{array}{llllllll}4 & 1 & 0.3036 & 0.221 & 1.0084 & 0.3061 & \mathbf{0 . 2 2 5}\end{array}$

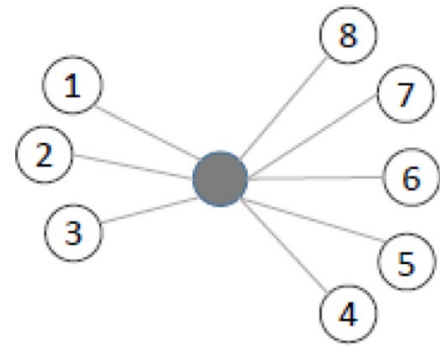

(a)

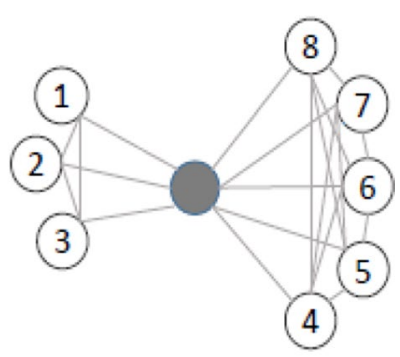

(b) Equilibrium

Fig. 6 Asymmetric agents: costly coordination. Parameter values: $S=5, q=0.1, \beta=1.2, \delta(A)=0.01$, $\delta(\mathrm{B})=0.01, \gamma=0.02, p=1.5$

\subsubsection{The Effect of Geographical Distance}

When the transportation of the resource is costly, the distance between the exploiting agent and the resource matters. Here, we explore the case where two of the agents are located further away, while the remaining six agents are located at an equal distance from the resource. More specifically, we assume that the distance between agent 1 and the resource is the longest $\left(l_{1}=0.5\right)$, followed by agent 2 who is located a bit closer $\left(l_{2}=0.3\right)$ and then the rest of the agents positioned even closer to the resource $\left(l_{i}=0.1, i=3, \ldots, 8\right)$.

The results, which correspond to the network configurations shown in Fig. 5, are presented in Table 3. More precisely, when the agents that are located closer to the resource create collaboration links, the profits of the unconnected agents decrease $\left(u_{i}^{(b)}<u_{i}^{(a)}\right.$, for 
$i=1,2)$, while the profits of the connected ones increase $\left(u_{i}^{(b)}>u_{i}^{(a)}\right.$, for $\left.i=3, \ldots, 8\right)$. In this case, the unconnected agents have an incentive to connect to the rest of the agents and enjoy higher profits, since $u_{i}^{(c)}>u_{i}^{(a),(b)}$, i.e., network (c) implies higher profits for all the agents. It is shown that the full connected network is also the optimal network structure.

Note that here we have assumed that any cost that is associated with the creation of collaboration links between the appropriators does not depend on the distance between them. If, however, connecting to a distant appropriator implies higher cost for the ones that are located closer to the resource (or closer to some other group of appropriators), then the incentives to collaborate become weaker. In such a case, we might observe the formation of subgroups, which is the case illustrated in Fig. 6. In particular, in the case where the benefits of collaboration are outweighed by the costs of connecting to some more distant appropriators, the agents find it profitable to connect only to nearby resource users. Thus, in our specific numerical example, we observe the formation of two subgroups: A (agents 1, 2 and 3) and B (agents 4, 5, 6, 7 and 8), which include agents that are located in the "neighborhood." The subgroup formation is more profitable for all the agents of the two groups $\left(u_{i}^{(b)}>u_{i}^{(a)}\right.$ in Table 4). In such a case, the planner should subsidize the "costly collaboration" in order to attain a full network of appropriators.

Note that the example of Fig. 6 can also illustrate the case where resource users form subgroups with people of the same ethnicity, as in Barnes et al. (2016a). Even though it is optimal to promote communication across segregated groups, which can prevent the overexploitation of natural resources, communication barriers between groups of different cultures impede the full network formation and lead to less sustainable environmental outcomes. Thus, understanding the social interactions across resource users and exploring the case-specific incentives to connect or not to other users can help the policy makers design policies that will secure the sustainable use of the natural resources.

\section{Conclusion}

In this paper, we use network theory to study the market and the optimal outcomes in a natural resource management problem. The externalities characterizing the exploitation of the resource under study, in the form of collaboration links, crowding effects, and resource stock effects, along with the conservation plan of the regulator, open the gap between decentralized and optimal harvesting. Different network structures have been studied and show how harvesting and aggregate welfare are affected. We also analyze the incentives of harvesting agents with respect to the formation or elimination of cooperative links, which determine the equilibrium network configurations. We show that homogeneous agents always have a strong incentive to move from an unconnected to a fully connected network, which is not necessarily the case when agents are heterogeneous.

Heterogeneity with respect to the distance of each agent to the resource, which implies higher transportation costs for the agents who are located further away, changes the harvesting amounts and the individual and aggregate payoffs. This also leads to different views regarding how profitable or not the cooperation between the harvesting agents is. We also show that the size of the firms matters. Thus, large appropriators, with lower extraction costs and advance technology, have weaker or no incentives to connect to the rest of the appropriators. Related work on fisheries has shown that acquiring and exchanging information between heterogeneous groups creates conflicting incentives. In fact, there are strong 
incentives for the withholding and strategic use of information and new knowledge, which is not likely to be shared because it can increase the fishing efficiency of others (Wilson 1990; Barnes et al. 2016b). It is thus important for decision makers to ensure that information is disseminated reaching different groups and that ties exist between all those resource users groups (Barnes et al. 2013).

Finally, in this paper, we show that the special nature of natural resources, when taken into account in the planner's problem, opens the gap between equilibrium and optimal harvesting. In particular, the more the regulator focuses on the conservation of the resource, the lower the optimal harvesting amount and the larger the gap between the market and the optimal harvesting. Moreover, our model helps us examine and determine the optimal structure of the resource network. When the optimal network structure has been identified, we will be able to design policies that are more in line with the scarce nature of the resources, prevent the overexploitation and promote the formation or the elimination of collaboration links between the appropriators. These policies can include both price-based instruments and direct regulations. Permits are already used in many countries for fishing and hunting. Our analysis suggests that collaborative behaviour among resource users should be (indirectly) subsidized, which could imply lower tax or permit price for collective action. Direct regulations were discussed above and refer to the efforts of decisions makers to ensure the existence of ties between different groups of resource users. Other ways of securing the sustainable use of the resource include monitoring and punishment by co-appropriators. Then, network analysis is used in order to describe the social ties among resource users who have the responsibility to monitor and punish each other in case of non-cooperative behaviour. According to the experimental literature, dense networks can prevent over-appropriation when such action is observed by everyone, but they can also encourage it when punishers struggle to coordinate and monitor non-cooperative behaviours (Shreedhar et al. 2020). Thus, identifying the efficient size and structure of the network is fundamental for the sustainable handling of resources.

The theoretical consideration of the impact of social networks in the management of natural resources can improve our understanding of the connection between social interactions and environmental outcomes. Thus, in view of a growing body of experimental and empirical literature on the impact of social interactions on large scale environmental outcomes, our paper can provide testable predictions that will facilitate the study of local interaction networks.

We recognize that the resource use is not a static problem. However, this is a first attempt to use the theory of network economics in order to study a natural resource management problem. Resource dynamics can be explicitly introduced in the problem by assuming, for example, that

$$
S_{t+1}-S_{t}=F\left(S_{t}\right)-\sum_{j=1}^{n} H_{j}(t), S_{0} \text { given, }
$$

where $F\left(S_{t}\right)$ is a standard resource-growth function. The network for the entire planning horizon can be regarded in this case as a multilayer network (e.g., Boccaletti et al. 2014). For the multilayer network, each time period $t \geq 0$ can be interpreted as representing a different network layer and each node on a layer can represent an appropriator at a given point in time who maximizes discounted profits. Intralayer connections are links among agents at a given point in time and can thus be interpreted as collaboration links as in the static network. Interlayer connections, on the other hand, follow the path of each harvester for $t \geq 0$. Severance of an interlayer connection for an agent $i$ can be interpreted as having the agent 
stop harvesting. The dynamic network problem can in principle be analyzed using dynamic optimization methods. The dynamic framework introduces new interesting issues which relate to the way that intralayer and interlayer links evolve in socially optimal solutions and market equilibrium. This undoubtedly is an interesting area for further research.

\section{Appendix A.1}

Proof of non-exhaustion We solve the maximization problem (3) by assuming that we have $n$ symmetric agents. The Lagrangian function of the first agent is stated as:

$$
\mathcal{L}=p H_{1}-\frac{1}{2} \frac{\beta}{(q S)^{2}} H_{1}^{2}-\gamma \sum_{j \neq 1}^{n} H_{1} H_{j}+\delta \sum_{j \neq 1}^{n} H_{1} H_{j}+\lambda\left(S-H_{1}-\sum_{j \neq 1}^{n} H_{j}\right)
$$

The necessary conditions are:

$$
\begin{aligned}
p-\frac{\beta}{(q S)^{2}} H_{1}-\gamma \sum_{j \neq 1}^{n} H_{j}+\delta \sum_{j \neq 1}^{n} H_{j}-\lambda \leq 0, & H_{1} \geqslant 0, & H_{1}\left(p-\frac{\beta}{(q S)^{2}} H_{1}-\gamma \sum_{j \neq 1}^{n} H_{j}+\delta \sum_{j \neq 1}^{n} H_{j}-\lambda\right)=0(\mathrm{NC} 1) \\
H_{1}+\sum_{j \neq 1}^{n} H_{j}-S \leq 0, & \lambda \geqslant 0, & \lambda\left(S-H_{1}-\sum_{j \neq 1}^{n} H_{j}\right)=0(\mathrm{NC} 2)
\end{aligned}
$$

Let NC1(i) denote the first condition on the first line etc.

NC1(iii): $H_{1}=0$ or $p-\frac{\beta}{(q S)^{2}} H_{1}-\gamma \sum_{j \neq 1}^{n} H_{j}+\delta \sum_{j \neq 1}^{n} H_{j}=\lambda$

Suppose $H_{1}=0$ (and by symmetry, $H_{2}=\cdots=H_{n}=0$ ). Then:

$$
\begin{aligned}
& \text { NC1(i): } p-\frac{\beta}{(q S)^{2}} H_{1}-\gamma \sum_{j \neq 1}^{n} H_{j}+\delta \sum_{j \neq 1}^{n} H_{j}-\lambda<0 \Rightarrow \\
& \quad p-\lambda<0 \Rightarrow 0<p<\lambda
\end{aligned}
$$

$\lambda>0$ means that NC2(i): $H_{1}+\sum_{j \neq 1}^{n} H_{j}-S=0$.But since $H_{i}=0$ for all $i$, then $S=0$, which is a contradiction because we have assumed that there is a positive stock of the resource. So, we have $H_{i}>0$ and $p-\frac{\beta}{(q S)^{2}} H_{1}-\gamma \sum_{j \neq 1}^{n} H_{j}+\delta \sum_{j \neq 1}^{n} H_{j}=\lambda$.

From NC2(iii): we have either $\lambda>0$ and $H_{1}+\sum_{j \neq 1}^{n} H_{j}-S=0$, or $\lambda=0$ and $H_{1}+\sum_{j \neq 1}^{n} H_{j}-S \leq 0$

$$
\text { If } \lambda>0 \text {, then } p-\frac{\beta}{(q S)^{2}} H_{1}-\gamma \sum_{j \neq 1}^{n} H_{j}+\delta \sum_{j \neq 1}^{n} H_{j}>0 \Rightarrow \underbrace{p}_{M R}>\underbrace{\frac{\beta}{(q S)^{2}} H_{1}+(\gamma-\delta) \sum_{j \neq 1}^{n} H_{j}}_{M C}
$$

Thus, $\lambda>0$ at the solution is not optimal because it implies that the marginal revenue is higher than the marginal cost of production (including the net congestion cost). So, we need to examine $\lambda=0$ at the solution. When $\lambda=0$ and $H>0$ : 
Table 5 Conditions for nonexhaustion in the full and star networks

\begin{tabular}{llll}
\hline $\begin{array}{l}\text { Number of } \\
\text { agents }\end{array}$ & 1 & $n$ & $n \rightarrow \infty$ \\
\hline Full & $S>\frac{\beta}{p q^{2}}$ & $S>\frac{\sqrt{4 \beta[(1-n) \gamma-\delta]+n^{2} p^{2} q^{2}}-n p q}{2 q(\gamma-\delta)(n-1)}$ & $S>\frac{p}{(\gamma-\delta)}$ \\
Star & $S>\frac{\beta}{p q^{2}}$ & $S>\frac{\sqrt{4 \beta(1-n) \gamma+n^{2} p^{2} q^{2}}-n p q}{2 q \gamma(n-1)}$ & $S>\frac{p}{\gamma}$ \\
\hline
\end{tabular}

NC1(ia): $p-\frac{\beta}{(q S)^{2}} H_{1}-\gamma \sum_{j \neq 1}^{n} H_{j}+\delta \sum_{j \neq 1}^{n} H_{j}=0$, in the full network.

NC1(ib): $p-\frac{\beta}{(q S)^{2}} H_{1}-\gamma \sum_{j \neq 1}^{n} H_{j}=0$, in the star network.

Also, when $\lambda=0, \mathrm{NC} 2(\mathrm{i}): H_{1}+\sum_{j \neq 1}^{n} H_{j}-S \leq 0$. Non-exhaustion implies that:

$$
N C 2(i): H_{1}+\sum_{j \neq 1}^{n} H_{j}-S<0
$$

Symmetry allows us to solve NC1(ia) [and NC1(ib)] wrt $H=H_{1}=\cdots=H_{i}$ for $n$ agents:

$$
\begin{gathered}
p-\frac{\beta}{(q S)^{2}} H-\gamma(n-1) H+\delta(n-1) H=0 \\
H=\frac{p}{\frac{\beta}{(q S)^{2}}+(n-1)(\gamma-\delta)}
\end{gathered}
$$

For $S>n H \Rightarrow S>\frac{n p}{\frac{\beta}{(q S)^{2}}+(n-1)(\gamma-\delta)} \Rightarrow \frac{p}{S}<\frac{\beta}{n(q S)^{2}}+\frac{(n-1)(\gamma-\delta)}{n}$.

If $n \rightarrow \infty$, then $\frac{p}{S}<(\gamma-\delta) \Rightarrow S>\frac{p}{(\gamma-\delta)}$. Table 5 summarizes the condition for nonexhaustion for a different number of appropriators in the two extreme network structures: the full and the star network.

We can show that $\frac{p}{(\gamma-\delta)}>\frac{\sqrt{4 \beta[(1-n) \gamma-\delta]+n^{2} p^{2} q^{2}}-n p q}{2 q(\gamma-\delta)(n-1)}$, which allows us to use the LHS of the inequality in Proposition 1.

\section{Appendix A.2}

\section{Nash equilibrium harvesting}

The matrix $\left[\frac{\beta-\gamma q^{2} S^{2}}{q^{2} S^{2}} \mathbf{I}+\gamma \mathbf{U}-\delta \mathbf{G}\right]$ in (7) is generically nonsingular and the equation (7) has a unique generic solution in $\mathbb{R}_{+}^{n}$, denoted by $\mathbf{H}^{*}$. Since $\mathbf{U}$ is an $n$-square matrix of ones, $\mathbf{U} \cdot \mathbf{H}^{*}=H^{*} \mathbf{1}$. Then,

$$
\begin{gathered}
{\left[\frac{\beta-\gamma q^{2} S^{2}}{q^{2} S^{2}} \mathbf{I}+\gamma \mathbf{U}-\delta \mathbf{G}\right] \cdot \mathbf{H}^{*}=p \cdot \mathbf{1}} \\
\frac{\beta-\gamma q^{2} S^{2}}{q^{2} S^{2}}\left[\mathbf{I}-\frac{\delta q^{2} S^{2}}{\beta-\gamma q^{2} S^{2}} \mathbf{G}\right] \cdot \mathbf{H}^{*}=\left(p-\gamma H^{*}\right) \cdot \mathbf{1}
\end{gathered}
$$


By inverting the matrix, we get:

$$
\begin{aligned}
\frac{\beta-\gamma q^{2} S^{2}}{q^{2} S^{2}} \mathbf{H}^{*} & =\left(p-\gamma H^{*}\right)\left[\mathbf{I}-\frac{\delta q^{2} S^{2}}{\beta-\gamma q^{2} S^{2}} \mathbf{G}\right]^{-1} \cdot \mathbf{1} \\
\frac{\beta-\gamma q^{2} S^{2}}{q^{2} S^{2}} \mathbf{H}^{*} & =\left(p-\gamma H^{*}\right) \mathbf{b}\left(\mathbf{g}, \frac{\delta q^{2} S^{2}}{\beta-\gamma q^{2} S^{2}}\right) \\
\mathbf{H}^{*}(\mathbf{\Sigma}) & =\frac{p}{\frac{\beta-\gamma q^{2} S^{2}}{q^{2} S^{2}}+\gamma b\left(\mathbf{g}, \frac{\delta q^{2} S^{2}}{\beta-\gamma q^{2} S^{2}}\right)} \mathbf{b}\left(\mathbf{g}, \frac{\delta q^{2} S^{2}}{\beta-\gamma q^{2} S^{2}}\right)
\end{aligned}
$$

\section{Appendix A.3}

\section{Aggregate harvesting}

To prove Proposition 2, let us assume that we have a partly connected network, g, where agents $i=1, \ldots, m$ are connected and $j=m+1, \ldots, n$ are unconnected. Then, from (8), we can calculate individual harvesting for two representative agents of the two groups, say agent 1 and $\quad m+1$. In particular, $H_{1}^{*}(\mathbf{g})=\frac{p}{\frac{\beta-\gamma q^{2} S^{2}}{q^{2} S^{2}}+\gamma b\left(\mathbf{g}, \frac{\delta q^{2} s^{2}}{\beta-\gamma q^{2} S^{2}}\right)} b_{1}\left(\mathbf{g}, \frac{\delta q^{2} S^{2}}{\beta-\gamma q^{2} S^{2}}\right) \quad$ and $H_{m+1}^{*}(\mathbf{g})=\frac{p}{\frac{\beta-\gamma q^{2} s^{2}}{q^{2} s^{2}}+\gamma b\left(\mathbf{g}, \frac{\delta q^{2} s^{2}}{\beta-\gamma q^{2} s^{2}}\right)}$, since the Bonacich centrality of the unconnected agent is equal to 1 , while $b_{1}\left(\mathbf{g}, \frac{\delta q^{2} S^{2}}{\beta-\gamma q^{2} S^{2}}\right)>1$. If agent $m+1$ now connects to agents $i=1, \ldots, m$, the two groups of connected and unconnected agents will be $i=1, \ldots, m+1$ and $j=m+2, \ldots, n$ respectively. This is a denser network, say $\mathbf{g}^{\prime}$, since agent $m+1$ is now connected to all agents $i=1, \ldots, m$. The new harvesting amounts of the two agents are now: $H_{1}^{*}\left(\mathbf{g}^{\prime}\right)=\frac{p}{\frac{\beta-\gamma q^{2} S^{2}}{q^{2} S^{2}}+\gamma b\left(\mathbf{g}^{\prime}, \frac{\delta q^{2} S^{2}}{\beta-\gamma q^{2} S^{2}}\right)} b_{1}\left(\mathbf{g}^{\prime}, \frac{\delta q^{2} S^{2}}{\beta-\gamma q^{2} S^{2}}\right) \quad$ and $\quad H_{m+1}^{*}\left(\mathbf{g}^{\prime}\right)=\frac{p}{\frac{\beta-\gamma q^{2} S^{2}}{q^{2} S^{2}}+\gamma b\left(\mathbf{g}^{\prime}, \frac{\delta q^{2} S^{2}}{\beta-\gamma q^{2} S^{2}}\right)}$ $b_{m+1}\left(\mathbf{g}^{\prime}, \frac{\delta q^{2} S^{2}}{\beta-\gamma q^{2} S^{2}}\right)$, which are clearly higher than before. The same is true for the sum of the associated harvesting amounts of the two networks: $\sum_{i} H_{i}^{*}\left(\mathbf{g}^{\prime}\right)>\sum_{i} H_{i}^{*}(\mathbf{g})$.

\section{Appendix A.4}

\section{Local Interactions Effect}

We prove that stronger local interactions increase the profits of connected agents (say $i$ ) and decrease the profits of unconnected agents ( a number of $N$ appropriators, of whom $n$ are connected to each other and $m$ are unconnected. The adjacency matrix $\mathbf{G}$ is:

$$
\mathbf{G}=\left[\begin{array}{ccccccccc}
0 & 1 & 1 & \ldots & 1 & 0 & 0 & \ldots & 0 \\
1 & 0 & 1 & \ldots & 1 & 0 & 0 & \ldots & 0 \\
1 & 1 & 0 & \ldots & 1 & 0 & 0 & \ldots & 0 \\
\ldots & \ldots & \ldots & \ldots & \ldots & \ldots & \ldots & \ldots & \ldots \\
1 & 1 & 1 & \ldots & 0 & 0 & 0 & \ldots & 0 \\
0 & 0 & 0 & \ldots & 0 & 0 & 0 & \ldots & 0 \\
0 & 0 & 0 & \ldots & 0 & 0 & 0 & \ldots & 0 \\
\ldots & \ldots & \ldots & \ldots & \ldots & \ldots & \ldots & \ldots & \ldots \\
0 & 0 & 0 & \ldots & 0 & 0 & 0 & \ldots & 0
\end{array}\right]
$$


The profits of the agents $i$ and $j$ are, then, given by:

$$
\begin{gathered}
\tilde{u}_{i}=\frac{\beta p^{2} q^{2} S^{2}\left(\beta-\gamma q^{2} S^{2}\right)^{2}}{2 \Delta^{2}} \\
\tilde{u}_{j}=\frac{\beta p^{2} q^{2} S^{2}\left(\beta-[\gamma-(n-1) \delta] q^{2} S^{2}\right)^{2}}{2 \Delta^{2}}
\end{gathered}
$$

where $\left.\Delta=\beta^{2}+\beta q^{2} S^{2}[(n+m-2) \gamma-(n-1) \delta]-\gamma q^{4} S^{4}[(n+m-1) \gamma+(n-1)(m-1) \delta)\right]>0$. Taking the derivative wrt $\delta$ :

$$
\frac{\partial \tilde{u}_{i}}{\partial \delta}=\frac{\beta p^{2} q^{4} S^{4}(n-1)\left(\beta-\gamma q^{2} S^{2}\right)^{2}\left(\beta+(m-1) \gamma q^{2} S^{2}\right)}{\Delta^{3}}>0,
$$

and

$$
\frac{\partial \tilde{u}_{j}}{\partial \delta}=-\frac{\beta \gamma p^{2} q^{6} S^{6} n(n-1)\left(\beta-\gamma q^{2} S^{2}\right)\left(\beta-[\gamma+(n-1) \delta] q^{2} S^{2}\right)}{\Delta^{3}}<0 .
$$

\section{Appendix A.5}

\section{Optimal Taxation, Bonacich Centrality}

We show how we derive the equilibrium harvesting amount under taxes in matrix form. The Nash equilibrium under a non-uniform tax solves:

$$
\begin{aligned}
-\boldsymbol{\Sigma} \cdot \mathbf{H}_{\tau}^{*} & =\left[\frac{\beta-\gamma q^{2} S^{2}}{q^{2} S^{2}} \mathbf{I}+\gamma \mathbf{U}-\delta \mathbf{G}\right] \cdot \mathbf{H}_{\tau}^{*}=p-\boldsymbol{\tau} \\
\boldsymbol{\tau} & =\left(\tau_{1}, \ldots \tau_{n}\right)
\end{aligned}
$$

Then,

$$
\begin{gathered}
{\left[\frac{\beta-\gamma q^{2} S^{2}}{q^{2} S^{2}} \mathbf{I}+\gamma \mathbf{U}-\delta \mathbf{G}\right] \cdot \mathbf{H}_{\tau}^{*}=p-\boldsymbol{\tau}} \\
\frac{\beta-\gamma q^{2} S^{2}}{q^{2} S^{2}}\left[\mathbf{I}-\frac{\delta q^{2} S^{2}}{\beta-\gamma q^{2} S^{2}} \mathbf{G}\right] \cdot \mathbf{H}_{\tau}^{*}=\left(p-\gamma H^{*}\right) \cdot \mathbf{1}-\boldsymbol{\tau}
\end{gathered}
$$

By inverting the matrix, we get:

$$
\begin{gathered}
\frac{\beta-\gamma q^{2} S^{2}}{q^{2} S^{2}} \mathbf{H}_{\tau}^{*}=\left(p-\gamma H^{*}\right)\left[\mathbf{I}-\frac{\delta q^{2} S^{2}}{\beta-\gamma q^{2} S^{2}} \mathbf{G}\right]^{-1} \cdot \mathbf{1}-\left[\mathbf{I}-\frac{\delta q^{2} S^{2}}{\beta-\gamma q^{2} S^{2}} \mathbf{G}\right]^{-1} \cdot \boldsymbol{\tau} \\
\frac{\beta-\gamma q^{2} S^{2}}{q^{2} S^{2}} \mathbf{H}_{\tau}^{*}=\left(p-\gamma H^{*}\right) \mathbf{b}\left(\mathbf{g}, \frac{\delta q^{2} S^{2}}{\beta-\gamma q^{2} S^{2}}\right)-\mathbf{b}_{\tau}\left(\mathbf{g}, \frac{\delta q^{2} S^{2}}{\beta-\gamma q^{2} S^{2}}\right) \\
{\left[\frac{\beta-\gamma q^{2} S^{2}}{q^{2} S^{2}}+\gamma b\left(\mathbf{g}, \frac{\delta q^{2} S^{2}}{\beta-\gamma q^{2} S^{2}}\right)\right] \mathbf{H}_{\tau}^{*}=p \mathbf{b}\left(\mathbf{g}, \frac{\delta q^{2} S^{2}}{\beta-\gamma q^{2} S^{2}}\right)-\mathbf{b}_{\tau}\left(\mathbf{g}, \frac{\delta q^{2} S^{2}}{\beta-\gamma q^{2} S^{2}}\right)}
\end{gathered}
$$




$$
\mathbf{H}_{\tau}^{*}(\boldsymbol{\Sigma})=\frac{1}{\frac{\beta-\gamma q^{2} S^{2}}{q^{2} S^{2}}+\gamma b\left(\mathbf{g}, \frac{\delta q^{2} S^{2}}{\beta-\gamma q^{2} S^{2}}\right)}\left[p \mathbf{b}\left(\mathbf{g}, \frac{\delta q^{2} S^{2}}{\beta-\gamma q^{2} S^{2}}\right)-\mathbf{b}_{\tau}\left(\mathbf{g}, \frac{\delta q^{2} S^{2}}{\beta-\gamma q^{2} S^{2}}\right)\right]
$$

where $\mathbf{b}_{\tau}\left(\mathbf{g}, \frac{\delta q^{2} S^{2}}{\beta-\gamma q^{2} S^{2}}\right)=\left[\mathbf{I}-\frac{\delta q^{2} S^{2}}{\beta-\gamma q^{2} S^{2}} \mathbf{G}\right]^{-1} \cdot \boldsymbol{\tau}=\mathbf{M}\left(\mathbf{g}, \frac{\delta q^{2} S^{2}}{\beta-\gamma q^{2} S^{2}}\right) \cdot \boldsymbol{\tau}$ is the weighted Bonacich centrality (look also at Remark 1 of Ballester et al. 2006).

Then the optimal tax should solve:

$$
\mathbf{H}_{\tau}^{*}=\mathbf{H}^{o} .
$$

Using the values for $\mathbf{H}^{*}, \mathbf{H}^{o}$, we obtain:

$$
\mathbf{H}^{*}-\mathbf{H}^{o}=\frac{\mathbf{M}\left(\mathbf{g}, \frac{\delta q^{2} S^{2}}{\beta-\gamma q^{2} S^{2}}\right) \cdot \boldsymbol{\tau}}{\frac{\beta-\gamma q^{2} S^{2}}{q^{2} S^{2}}+\gamma b\left(\mathbf{g}, \frac{\delta q^{2} S^{2}}{\beta-\gamma q^{2} S^{2}}\right)}
$$

and finally the vector of the optimal tax is:

$$
\boldsymbol{\tau}=\left[\frac{\beta-\gamma q^{2} S^{2}}{q^{2} S^{2}}+\gamma b\left(\mathbf{g}, \frac{\delta q^{2} S^{2}}{\beta-\gamma q^{2} S^{2}}\right)\right]\left[\mathbf{M}\left(\mathbf{g}, \frac{\delta q^{2} S^{2}}{\beta-\gamma q^{2} S^{2}}\right)\right]^{-1}\left(\mathbf{H}^{*}-\mathbf{H}^{o}\right) .
$$

\section{Appendix A.6}

\section{Costly Transportation}

We show how we get the equilibrium harvesting amount in matrix form.

By inverting the matrix (46), we get:

$$
\begin{gathered}
\frac{\beta-\gamma q^{2} S^{2}}{q^{2} S^{2}} \mathbf{H}^{*}=\left[\mathbf{I}-\frac{\delta q^{2} S^{2}}{\beta-\gamma q^{2} S^{2}} \mathbf{G}\right]^{-1} \hat{\mathbf{p}}-\gamma H^{*}\left[\mathbf{I}-\frac{\delta q^{2} S^{2}}{\beta-\gamma q^{2} S^{2}} \mathbf{G}\right]^{-1} \cdot \mathbf{1} \\
\frac{\beta-\gamma q^{2} S^{2}}{q^{2} S^{2}} \mathbf{H}^{*}=\mathbf{b}_{\hat{\mathbf{p}}}\left(\mathbf{g}, \frac{\delta q^{2} S^{2}}{\beta-\gamma q^{2} S^{2}}\right)-\gamma H^{*} b\left(\mathbf{g}, \frac{\delta q^{2} S^{2}}{\beta-\gamma q^{2} S^{2}}\right) \\
{\left[\frac{\beta-\gamma q^{2} S^{2}}{q^{2} S^{2}}+\gamma b\left(\mathbf{g}, \frac{\delta q^{2} S^{2}}{\beta-\gamma q^{2} S^{2}}\right)\right] \mathbf{H}^{*}=\mathbf{b}_{\hat{\mathbf{p}}}\left(\mathbf{g}, \frac{\delta q^{2} S^{2}}{\beta-\gamma q^{2} S^{2}}\right)} \\
\mathbf{H}^{*}(\mathbf{\Sigma})=\frac{1}{\frac{\beta-\gamma q^{2} S^{2}}{q^{2} S^{2}}+\gamma b\left(\mathbf{g}, \frac{\delta q^{2} S^{2}}{\beta-\gamma q^{2} S^{2}}\right)} \mathbf{b}_{\hat{\mathbf{p}}}\left(\mathbf{g}, \frac{\delta q^{2} S^{2}}{\beta-\gamma q^{2} S^{2}}\right)
\end{gathered}
$$

\section{Appendix A.7}

The size of firms

In order to solve the problem (50), we define the matrix $\mathbf{X}$ such that $\mathbf{B}=\mathbf{X}+\mathbf{I}$. Then, 


$$
\begin{gathered}
-\boldsymbol{\Sigma} \cdot \mathbf{H}=\left[\left(\frac{1}{q^{2} S^{2}}\right)(\mathbf{X}+\mathbf{I})-\gamma \mathbf{I}+\gamma \mathbf{U}-\delta \mathbf{G}\right] \cdot \mathbf{H}=p \cdot \mathbf{1} . \\
{\left[\left(\frac{1}{q^{2} S^{2}}\right) \mathbf{X}+\left(\frac{1}{q^{2} S^{2}}-\gamma\right)\left[\mathbf{I}-\frac{\delta q^{2} S^{2}}{1-\gamma q^{2} S^{2}} \mathbf{G}\right]\right] \cdot \mathbf{H}=\left(p-\gamma H^{*}\right) \cdot \mathbf{1}}
\end{gathered}
$$

Next, we define $\mathbf{M}(\mathbf{g}, a)=\left[\mathbf{I}-\frac{\delta q^{2} S^{2}}{1-\gamma q^{2} S^{2}} \mathbf{G}\right]$ and multiply (7.2) by $\mathbf{M}^{-1}$ to obtain:

$$
\begin{gathered}
{\left[\left(\frac{1}{q^{2} S^{2}}\right) \mathbf{M}^{-1} \mathbf{X}+\left(\frac{1}{q^{2} S^{2}}-\gamma\right)\right] \cdot \mathbf{H}=\left(p-\gamma H^{*}\right) \cdot \mathbf{b}\left(\mathbf{g}, \frac{\delta q^{2} S^{2}}{1-\gamma q^{2} S^{2}}\right)} \\
{\left[\left(\frac{1}{q^{2} S^{2}}\right) \mathbf{M}^{-1} \mathbf{X}+\left(\frac{1}{q^{2} S^{2}}-\gamma\right)+\gamma H^{*} b\left(\mathbf{g}, \frac{\delta q^{2} S^{2}}{1-\gamma q^{2} S^{2}}\right)\right] \cdot \mathbf{H}=p \mathbf{b}\left(\mathbf{g}, \frac{\delta q^{2} S^{2}}{1-\gamma q^{2} S^{2}}\right)} \\
{\left[\left(\frac{1}{q^{2} S^{2}}\right) \mathbf{M}^{-1} \mathbf{X}+\left(\left(\frac{1}{q^{2} S^{2}}-\gamma\right)+\gamma H^{*} b\right) \mathbf{I}\right] \cdot \mathbf{H}=p \mathbf{b}\left(\mathbf{g}, \frac{\delta q^{2} S^{2}}{1-\gamma q^{2} S^{2}}\right)}
\end{gathered}
$$

If we define the matrix $\Phi=\left[\left(\frac{1}{q^{2} S^{2}}\right) \mathbf{M}^{-1} \mathbf{X}+\left(\left(\frac{1}{q^{2} S^{2}}-\gamma\right)+\gamma H^{*} b\right) \mathbf{I}\right]$, the equilibrium harvesting becomes:

$$
\mathbf{H}^{*}=p \Phi^{-1} \mathbf{b}\left(\mathbf{g}, \frac{\delta q^{2} S^{2}}{1-\gamma q^{2} S^{2}}\right) .
$$

Funding Open access funding provided by Swedish University of Agricultural Sciences.

Open Access This article is licensed under a Creative Commons Attribution 4.0 International License, which permits use, sharing, adaptation, distribution and reproduction in any medium or format, as long as you give appropriate credit to the original author(s) and the source, provide a link to the Creative Commons licence, and indicate if changes were made. The images or other third party material in this article are included in the article's Creative Commons licence, unless indicated otherwise in a credit line to the material. If material is not included in the article's Creative Commons licence and your intended use is not permitted by statutory regulation or exceeds the permitted use, you will need to obtain permission directly from the copyright holder. To view a copy of this licence, visit http://creativecommons.org/licenses/by/4.0/.

\section{References}

Agrawal A, Gibson C (1999) Community and conservation: beyond enchantment and disenchantment. World Dev 27:629-649

Allouch N (2015) On the private provision of public goods on networks. J Econ Theory 157:527-552

Ballester C, Calvó-Armengol A, Zenou Y (2006) Who's who in networks. Wanted: the key player. Econometrica 74(5):1403-1417

Barnes ML, Arita S, Allen SD, Gray SA, Leung PS (2013) The influence of ethnic diversity on social network structure in a common-pool resource system: implications for collaborative management. Ecol Soc 18(1):23

Barnes ML, Lynham J, Kalberg K, Leung P (2016a) Social networks and environmental outcomes. Proc Natl Acad Sci 113(23):6466-6471

Barnes ML, Kalberg K, Pan M, Leung P (2016b) When is brokerage negatively associated with economic benefits? Ethnic diversity, competition, and common-pool resources. Soc Netw 45:55-65 
Boccaletti S, Bianconi G, Criado R, del Genio CI, Gómez-Gardeñes J, Romance M, Sendiña-Nadal I, Wang Z, Zanin M (2014) The structure and dynamics of multilayer networks. Phys Rep 544(1):1-122

Bodin Ö, Crona BI (2008) Management of natural resources at the community level: exploring the role of social capital and leadership in a rural fishing community. World Dev 36(12):2763-2779

Bodin Ö, Crona BI (2009) The role of social networks in natural resource governance: what relational patterns make a difference? Global Environ Change 19(3):366-374

Bodin Ö, Prell C (2011) Social networks and natural resource management: uncovering the social fabric of environmental governance. Cambridge University Press, Cambridge

Bonacich P (1987) Power and centrality: a family of measures. Am J Soc 92:1170-1182

Bramoullé Y, Kranton R (2007) Public goods in networks. J Econ Theory 135:478-494

Carlsson L, Berkes F (2005) Co-management: concepts and methodological implications. J Environ Manag 75:65-76

Carlsson L, Sandström A (2008) Network governance of the commons. Int J Commons 2(1):33-54

Cavalcanti C, Schläpfer F, Schmid B (2010) Public participation and willingness to cooperate in common-pool resource management: a field experiment with fishing communities in Brazil. Ecol Econ 69:613-622

Cavalcanti C, Engel S, Leibbrandt A (2013) Social integration, participation, and community resource management. J Environ Econ Manag 65:262-276

Conley T, Udry C (2001) Social learning through networks: the adoption of new agricultural technologies in Ghana. Am J Agric Econ 83(3):668-673

Crona B, Bodin Ö (2006) WHAT you know is WHO you know? Communication patterns among resource users as a prerequisite for co-management. Ecol Soc 11(2):7

Crona B, Bodin Ö (2011) Friends or neighbors? Subgroup heterogeneity and the importance of bonding and bridging ties in natural resource governance. In Ö Bodin \& C. Prell (Eds.), Social Networks and Natural Resource Management: Uncovering the Social Fabric of Environmental Governance (pp. 206-233). Cambridge: Cambridge University Press. https://doi.org/10.1017/CBO9780511 894985.010

Currarini S, Marchiori C, Tavoni A (2016) Network economics and the environment: insights and perspectives. Environ Resour Econ 65(1):159-189

Delgado-Serrano MM (2017) Trade-offs between conservation and development in community-based management initiatives. Int J Commons 11(2):969-991

Fischbacher U, Gächter S, Fehr E (2001) Are people conditionally cooperative? Evidence from a public goods experiment. Econ Lett 71(3):397-404

Gangadharan L, Nikiforakis N, Villeval MC (2017) Normative conflict and limits of self-governance in heterogeneous populations. Eur Econ Rev 100:143-156

Günther M, Hellmann T (2017) International environmental agreements for local and global pollution. J Environ Econ Manag 81:38-58

Gray S, Shwom R, Jordan R (2012) Understanding factors that influence stakeholder trust of natural resource science and institutions. Environ Manag 49(3):663-674

Hackett S, Edella S, Walker J (1994) The role of communication in resolving commons dilemmas: experimental evidence with heterogeneous appropriators. J Environ Econ Manag 27:99-126

Helsley R, Zenou Y (2014) Social networks and interactions in cities. J Econ Theory 150:426-466

İlkiliç R (2011) Networks of common property resources. Econ Theory 47:105-134

Ioannides YM (2012) From neighborhood to nations: the economics of social interactions. Princeton University Press, Princeton

Jackson MO (2014) Networks in the understanding of economic behaviors. J Econ Perspect 28:3-22

Jackson M, Watts A (2002) The evolution of social and economic networks. J Econ Theory 106:265-295

Jackson M, Wolinsky A (1996) A strategic model of social and economic networks. J Econ Theory $71: 44-74$

Jackson M, Zenou Y (2015) Games on networks. In: Young P, Zamir S (eds) Handbook of game theory, vol 4. Elsevier, Amsterdam, pp 91-157

Jackson MO, Rogers BW, Zenou Y (2017) The economic consequences of social network structure. J Econ Lit 55(1):49-95

Kubo H, Supriyanto B (2010) From fence-and-fine to participatory conservation: mechanisms of transformation in conservation governance at the Gunung Halimun-Salak National Park, Indonesia. Biodivers Conserv 19:1785-1803

Lacomba JA, Lagos F, Perote J (2017) The Lazarillo's game: sharing resources with asymmetric conditions. PLoS ONE 12:e0180421 
Layzer JA (2008) Natural experiments: ecosystem-based management and the environment. MIT Press, Cambridge

Nygren A (2005) Community-based forest management within the context of institutional decentralization in Honduras. World Dev 33:639-655

Ostrom E (1990) Governing the commons: the evolution of institutions for collective action. Cambridge University Press, New York

Ostrom E (2004) Understanding collective action. In: Meinzen-Dock R, Di Gregorio M (eds) Collective action and property rights for sustainable development, 2020 vision for food, agriculture and the environment. Focus 11, IFPRI International Food Policy Research Institute, Washington

Pomeroy R, Parks J, Pollnac R, Campson T, Genio E, Marlessy C, Holle E, Pido M, Nissapa A, Boromthanarat S, Nguyen TH (2007) Fish wars: conflict and collaboration in fisheries management in Southeast Asia. Mar Policy 31(6):645-656

Ramirez-Sanchez S (2011) Who and how: engaging well-connected fishers in social networks to improve fisheries management and conservation. Social Networks and Natural Resource Management: Uncovering the Fabric of Environmental Governance, ed. Bodin and C. Prell. Cambridge University Press, pp 119-146

Scullion JJ, Vogt KA, Winkler-Schor S, Sienkiewicz A, Pena C, Hajek F (2016) Designing conservationdevelopment policies for the forest frontier. Sustain Sci 11:295-306

Shreedhar G, Tavoni A, Marchiori C (2020) Monitoring and punishment networks in an experimental common pool resource dilemma. Environ Dev Econ 25:66-94

Smith VL (1968) Economics of production from natural resources. Am Econ Rev 58(3):409-431

Smith VL (1969) On models of commercial fishing. J Polit Econ 77(2):181-198

Tembata K, Takeuchi K (2018) Collective decision making under drought: an empirical study of water resource management in Japan. Water Resour Econ 22:19-31

Valente TW (2012) Network interventions. Science 337(6090):49-53

Verdier T, Zenou Y (2017) The role of social networks in cultural assimilation. J Urban Econ 97:15-39

Watts A (2001) A dynamic model of network formation. Games Econ Behav 34:331-341

Wilson JA (1990) Fishing for knowledge. Land Econ 66(1):12-29

Publisher's Note Springer Nature remains neutral with regard to jurisdictional claims in published maps and institutional affiliations. 